Article

\title{
Transcriptomic and Physiological Responses to Oxidative Stress in a Chlamydomonas reinhardtii Glutathione Peroxidase Mutant
}

\author{
Xiaocui Ma ${ }^{1,3}$, Baolong Zhang ${ }^{1,2,3}$, Rongli Miao ${ }^{1,2,3}$, Xuan Deng ${ }^{1,2}$, You Duan ${ }^{1,3}$, \\ Yingyin Cheng ${ }^{1}$, Wanting Zhang ${ }^{1}$, Mijuan Shi ${ }^{1}$, Kaiyao Huang ${ }^{1,2, *}$ and Xiao-Qin Xia ${ }^{1,3, *(D)}$ \\ 1 Institute of Hydrobiology, Chinese Academy of Sciences, Wuhan 430072, Hubei, China; \\ maxiaocui_luck@126.com (X.M.); zhangbl@ihb.ac.cn (B.Z.); mronli@ihb.ac.cn (R.M.); \\ dengxuan@ihb.ac.cn (X.D.); duanyou@outlook.com (Y.D.); cyy@ihb.ac.cn (Y.C.); \\ zhangwanting@ihb.ac.cn (W.Z.); shimijuan@ihb.ac.cn (M.S.) \\ 2 Key Laboratory of Algal Biology, Institute of Hydrobiology, Chinese Academy of Sciences, \\ Wuhan 430072, Hubei, China \\ 3 University of Chinese Academy of Sciences, Beijing 100039, China \\ * Correspondence: huangky@ihb.ac.cn (K.H.); xqxia@ihb.ac.cn (X.-Q.X.)
}

Received: 13 March 2020; Accepted: 21 April 2020; Published: 24 April 2020

\begin{abstract}
Aerobic photosynthetic organisms such as algae produce reactive oxygen species (ROS) as by-products of metabolism. ROS damage biomolecules such as proteins and lipids in cells, but also act as signaling molecules. The mechanisms that maintain the metabolic balance in aerobic photosynthetic organisms and how the cells specifically respond to different levels of ROS are unclear. Glutathione peroxidase (GPX) enzymes detoxify hydrogen peroxide or organic hydroperoxides, and thus are important components of the antioxidant system. In this study, we employed a Chlamydomonas reinhardtii glutathione peroxidase knockout (gpx5) mutant to identify the genetic response to singlet oxygen $\left({ }^{1} \mathrm{O}_{2}\right)$ generated by the photosensitizer rose bengal (RB). To this end, we compared the transcriptomes of the parental strain CC4348 and the gpx5 mutant sampled before, and $1 \mathrm{~h}$ after, the addition of RB. Functional annotation of differentially expressed genes showed that genes encoding proteins related to ROS detoxification, stress-response-related molecular chaperones, and ubiquitin-proteasome pathway genes were upregulated in CC4338. When GPX5 was mutated, higher oxidative stress specifically induced the TCA cycle and enhanced mitochondrial electron transport. Transcription of selenoproteins and flagellar-associated proteins was depressed in CC 4348 and the gpx 5 mutant. In addition, we found iron homeostasis played an important role in maintaining redox homeostasis, and we uncovered the relationship between ${ }^{1} \mathrm{O}_{2}$ stress and iron assimilation, as well as selenoproteins. Based on the observed expression profiles in response to different levels of oxidative stress, we propose a model for dose-dependent responses to different ROS levels in Chlamydomonas.
\end{abstract}

Keywords: glutathione peroxidase; oxidative stress; RNA-seq; reactive oxygen species; singlet oxygen; Chlamydomonas reinhardtii

\section{Introduction}

Plants and algae are the main producers of atmospheric oxygen, which inevitably leads to the production of a large amount of reactive oxygen species (ROS) within cells. Photosystem releases oxygen with $\mathrm{H}_{2} \mathrm{O}$, accompanied by the production of ROS, which include singlet oxygen, superoxide, hydrogen peroxide, and hydroxyl radicals [1-3]. ROS play a dual role in plant physiological processes, potentially causing damage (membrane lipid peroxidation, DNA damage, and irreversible hyperoxidation of 
proteins) but also functioning as indispensable short- and long-range signaling molecules in numerous cellular responses $[4,5]$. In vascular plants, ROS are involved in the regulation of development, cell differentiation, intracellular redox levels, signal transduction, stress responses, and induction of apoptosis [4,6-10]. Therefore, ROS homeostasis must be carefully controlled to support signaling while limiting damage to cells.

To protect themselves from damage caused by oxidative stress and to redirect the actions of ROS into signaling pathways, plants have evolved a diverse set of antioxidant factors, including enzymes that detoxify ROS. For example, the non-selenocysteine glutathione peroxidase GPX5 detoxifies hydrogen peroxide with cytosolic thioredoxin TRX- $h 1$, not glutathione (GSH), as the electron donor $[11,12]$. In Chlamydomonas reinhardtii, singlet oxygen $\left({ }^{1} \mathrm{O}_{2}\right)$ produced from the photosensitizers neutral red, methylene blue, or rose bengal (RB) induces a remarkable upregulation of GPX5 expression. Hydrogen peroxide or the superoxide-generating compound paraquat induces a somewhat weaker upregulation of GPX5 [11,13,14]. A C. reinhardtii gpx5 knockout mutant showed depressed synthesis of lipid droplets compared with its parental strain CC4348 [15], suggesting that a high level of ROS attenuated lipid-droplet formation.

Research in algae and vascular plants has investigated the signaling pathways that modulate gene expression in response to ${ }^{1} \mathrm{O}_{2}$ stress, including direct and retrograde (i.e., chloroplast-to-nucleus) signaling. For example, the cytosolic zinc finger protein METHYLENE BLUE SENSITIVITY (MBS) is conserved in Arabidopsis and Chlamydomonas and affects the oxidative stress response [16]. Screening for Chlamydomonas lines that lack the acclimation response to ${ }^{1} \mathrm{O}_{2}$ identified the singlet oxygen acclimation knocked-out 1 (sak1) mutant [17]. SAK1 is a putative transcription factor and is hyperphosphorylated during ${ }^{1} \mathrm{O}_{2}$ acclimation. How these signaling pathways act together to modulate the response to ${ }^{1} \mathrm{O}_{2}$ stress remains unclear.

In this study, we employed the unicellular green alga C. reinhardtii to investigate ${ }^{1} \mathrm{O}_{2}$ signaling and responses to oxidative stress in the gpx5 mutant. To this end, we sequenced the transcriptomes of the parental C. reinhardtii strain CC4348, the gpx 5 mutant, and the complemented strain L27 before and after treatment with RB. By comparing the transcriptomes, we elucidated how ${ }^{1} \mathrm{O}_{2}$ signaling regulates gene transcription and the role of the key antioxidant enzyme, GPX5, in this response.

\section{Materials and Methods}

\subsection{Strains and Culture Conditions}

The parental strain of $C$. reinhardtii was CC4348, which was also available as sta6 (cw15 nit1 NIT2 arg7-7 sta6-1:ARG7 $\mathrm{mt}^{+}$) strain descripted as from the Chlamydomonas Resource Center (http: //www.chlamycollection.org). The sta6 mutant has been shown not only defective for the small subunit of the heterotetrameric ADP-glucose pyrophosphorylase [18,19], being also affected in respiratory burst oxidase gene (RBO1, Cre03.g188300) [20] and sensitive to oxidative stress levels of lipid peroxidation [21]. Because of high oil accumulation under N-starvation, CC4348 was initially used to screen the mutants with defects in lipid droplets formation, the gpx 5 mutant was one of them. The gpx5 mutant and its complemented strain L27 were obtained as described previously [15]. As the initial concentration at 1.7 $\times 10^{6}$ cells $\cdot \mathrm{mL}^{-1}\left(\mathrm{OD}_{750}=0.5\right)$, all cells were cultured mixotrophically in Tris-acetate-phosphate (TAP) medium ( $20 \mathrm{mM}$ Tris, $0.4 \mathrm{mM} \mathrm{MgSO}_{4} \cdot 7 \mathrm{H}_{2} \mathrm{O}, 0.34 \mathrm{mM} \mathrm{CaCl}_{2} \cdot 2 \mathrm{H}_{2} \mathrm{O}, 10 \mathrm{mM} \mathrm{NH}_{4} \mathrm{Cl}, 1 \mathrm{mM}$ phosphate, $10 \mathrm{~mL} / \mathrm{L}$ glacial acetic acid and trace elements [22]) at $25^{\circ} \mathrm{C}$ under constant incident illumination of $60 \mu \mathrm{mol} \cdot \mathrm{m}^{-2} \cdot \mathrm{s}^{-1}$ with continuous shaking at $120 \mathrm{rpm}$. Cells were grown on solidified TAP medium containing $1.5 \%$ agar at $25^{\circ} \mathrm{C}$ under constant incident illumination of $60 \mu \mathrm{mol} \cdot \mathrm{m}^{-2} \cdot \mathrm{s}^{-1}$ for about 5 days.

\subsection{RB treatment Assays and Sampling}

For the RB treatments, $100 \mathrm{~mL}$ of liquid culture was harvested at a density of $2 \times 10^{6}$ cells $\mathrm{mL}^{-1}$ (about $\mathrm{OD}_{750}=0.6$ ) by centrifugation at $2500 \times g$ for $3 \mathrm{~min}$ at room temperature. The supernatant was discarded, the cells were washed in fresh TAP medium one time, and then they were resuspended in 
an equal volume of fresh culture medium and grown at $25{ }^{\circ} \mathrm{C}$ under constant incident illumination of $60 \mu \mathrm{mol} \cdot \mathrm{m}^{-2} \cdot \mathrm{s}^{-1}$ with continuous shaking at $120 \mathrm{rpm}$, and fresh RB (Sigma Aldrich, Missouri, USA) was added to a final concentration of $1 \mu \mathrm{M}$. Samples taken immediately upon inoculation with RB-containing media were labeled as $0 \mathrm{~min}$, and subsequent samples were taken after 15, 30, 45, and $60 \mathrm{~min}$ incubation and labeled accordingly. Only samples at 0, 30, and 60 min were used for RNA-seq, while all were used for validation by quantitative real-time PCR. This experiment was performed in triplicate.

\subsection{ROS Measurement}

Cells were stained with $2^{\prime}, 7^{\prime}$-dichlorofluorescein diacetate (DCFH-DA) (Sigma Aldrich, Missouri, USA) as described in our previous study [15]. The nonpolar, nonionic DCFH-DA becomes nonfluorescent DCFH after crossing cell membranes and being enzymatically hydrolyzed by intracellular esterases. Hydrogen peroxide oxidizes DCFH to fluorescent dichlorofluorescein (DCF). The fluorescence of DCF was detected by using a multi-mode microplate reader (Filter Max F5, Molecular Devices; excitation at $485 \mathrm{~nm}$ and emission at $530 \mathrm{~nm}$ ). The ROS abundance after treatment with RB was quantified by the following equation: $\left(\mathrm{F}_{\mathrm{TAP}+\mathrm{RB}}\right) / \mathrm{F}_{\mathrm{TAP}}$. Under oxidative stress condition, the level of ROS was normalized for each strain from untreated condition independently.

\subsection{Isolation of Total RNA}

Ten $\mathrm{mL}$ sample cultures (at a density of $2 \times 10^{6}$ cells $\mathrm{ml}^{-1}$ ) were collected by centrifugation for $3 \mathrm{~min}$ at $2500 \times \mathrm{g}$ and $4{ }^{\circ} \mathrm{C}$, washed in RNase-free water, and resuspended in $5 \mathrm{~mL}$ of Trizol Reagent (Invitrogen, CA, USA). The suspension was rocked for $5 \mathrm{~min}$ before it was flash-frozen in liquid nitrogen and stored at $-80^{\circ} \mathrm{C}$. After all samples were collected, the samples resuspended in Trizol were taken out, thawed at $24^{\circ} \mathrm{C}$ for about $10 \mathrm{~min}$. In proportion to the volume of Trizol, $1 \mathrm{~mL}$ of chloroform was added to the suspension, rocked for $15 \mathrm{~s}$, following by maintaining on ice for $3 \mathrm{~min}$. After centrifuged at $12,000 \times \mathrm{g}$ for $15 \mathrm{~min}$ at $4{ }^{\circ} \mathrm{C}$, the supernatant was saved to add $2.5 \mathrm{~mL}$ of isopropanol, followed by maintaining on ice for $10 \mathrm{~min}$. After centrifuged at $12,000 \times \mathrm{g}$ for $10 \mathrm{~min}$ at $4{ }^{\circ} \mathrm{C}$, the supernatant was washed by $75 \%$ ethanol, dried, and dissolved in diethyl pyrocarbonate-treated double-distilled $\mathrm{H}_{2} \mathrm{O}\left(\mathrm{ddH}_{2} \mathrm{O}\right)$. After removal of the final traces of ethanol, the pellet was resuspended in $50 \mu \mathrm{L}$ of $\mathrm{H}_{2} \mathrm{O}$ containing $0.1 \%$ diethyl pyrocarbonate. The RNA concentration was measured on a NanoDrop 2000 spectrophotometer (Thermo Fisher Scientific, MA, USA), and the quality of the RNA was assessed on an Agilent 2100 Bioanalyzer (Agilent Technologies, CA, USA).

To analyze the gene expression of the whole-genome, RNA samples from CC4348, the gpx5 mutant, and the complemented strain L27 were isolated and used to construct the sequencing libraries for Illumina sequencing. Briefly, mRNA was purified from $5 \mu \mathrm{g}$ of total RNA using oligo (dT) magnetic beads, broken into 450-550 bp fragments, and added unique adapter for every sample, using the TruSeq RNA sample preparation kit (Illumina, CA, USE) according to the manufacturer's instructions.

\subsection{RNA-Sequencing, Mapping, and Gene Expression Analyses}

For estimating the transcript abundance in the RB incubation time-course experiments, the pooled sequencing libraries were sequenced on the Illumina HiSeq X-ten platform (Illumina Inc., CA, USA) for $150 \mathrm{bp}$ pair end sequencing mode. About $10 \mathrm{G}$ of sequencing data were obtained per library for each sample. After filtering low-quality reads with a Perl script (IlluQC.pl) from the NGSQC Toolkit [23], high-quality reads were mapped to the C. reinhardtii version 5.5 genome (Department of Energy JGI), using TopHat software [24]. No more than two mismatches per sequencing read were allowed. More than 10 million reads with unique mapping were mapped for each sample, and the uniquely mapped ratio was about $90 \%$ of total mapped reads in each sample. Unique mapping reads were assigned to $C$. reinhardtii version 5.5 transcripts, to record the mapped count number, using HT-Seq [25]. Analyses of differential expression, including FDR calculations, were performed by using the Bioconductor package edgeR [26]. Expression estimates were in units of RPKM [27] normalized 
by library size and mappable transcript length. If the RPKM of one transcript was equal to zero, the minimum value in that experiment was used to replace it. The sets of genes that met the following three conditions in each comparison were selected for further analysis: (1) RPKM $\log _{2}$ ratios were considered significant if $\geq 2$ or $\leq-2$; (2) at least one $R P K M \geq 10$; (3) the $p$-value for differential expression was set to $\leq 0.05$.

\subsection{Quantitative Real-Time PCR}

RNA was isolated from samples after treatment with $1 \mu \mathrm{M}$ RB immediately ( 0 min) and after 15 , 30, 45, and $60 \mathrm{~min}$. The cDNA was transcribed by using First-Strand cDNA transcription kit (Thermo Fisher Scientific, MA, USA) according to the manufacturer's protocol. The cDNA was then diluted 1:3. Quantitative real-time PCR (qRT-PCR) was performed, using specific primers (Table S7) and LightCycler 480 SYBR Green (Applied Biosystems, CA, USA) according to manufacturer's protocol on the Applied Biosystems 7500 Fast real-time PCR system. The sizes of amplification products were 100 to $300 \mathrm{bp}$. All samples were run in duplicate, with CT value normalized to C $\beta L P$. The $2^{-\Delta \Delta C T}$ method [28] was employed to calculate relative fold differences.

\subsection{Measurements of Photosynthetic Efficiency}

Photosynthetic efficiency was measured as the PSII maximum quantum yield, using the Fv/Fm parameter. Then, $3 \mathrm{~mL}$ of culture, at a density of $1 \times 10^{6}$ cells $/ \mathrm{mL}$, was collected and dark-adapted for $15 \mathrm{~min}$. Fv/Fm was measured by using a multiple excitation wavelength modulated chlorophyll fluorometer (Heinz Walz Gmbh Effeltrich, Wurzburg, Germany).

\subsection{Measurements of Chlorophyll Content}

For chlorophyll content, including chlorophyll $a$ and chlorophyll $b, 4 \times 10^{6}$ cells were collected into a $2 \mathrm{~mL}$ centrifuge tube and centrifuged at 12,500 $\mathrm{g}$ for $1 \mathrm{~min}$. The precipitate was dissolved in $1 \mathrm{~mL}$ of methanol for $24 \mathrm{~h}$ in darkness, at $4{ }^{\circ} \mathrm{C}$. After centrifugation at $12,500 \times g$ for $2 \mathrm{~min}, 750 \mu \mathrm{L}$ of supernatant was used to measure absorbance values at 665.2 and $652.0 \mathrm{~nm}$, using a spectrophotometer. The chlorophyll content was determined according to the following equation [29]: chlorophyll content $(\mu \mathrm{g} / \mathrm{mL})=2.71 \times \mathrm{OD}_{665.2}+22.12 \times \mathrm{OD}_{652.0}$.

\subsection{Measurements of Percentage of Cells with Flagella and Flagella Length}

CC4348 and its derivative gpx5 mutant lack a cell wall and are difficult to be crossed to obtain the cell with flagella, so widely used wild-type CC125 strain was employed for measuring flagella length. After treatment with $1 \mu \mathrm{M}$ RB for $30 \mathrm{~min}$, aliquots of CC125 cells were fixed with $0.5 \%$ Lugol's solution. Images were recorded with a microscope (Nikon ECLIPSE Ti-U, Tokyo, Japan) equipped with a camera (Digital Sight DS-U3, Nikon, Tokyo, Japan) with an LWD 40× objective lens. At least 100 flagella were measured, and 200 cells were counted, and the ratio of the ciliated cells was calculated, using the NIS-Elements BR (version 4.10) software. This experiment was performed in triplicate.

\section{Results}

\subsection{GPX5 Maintains ROS Homeostasis in C. reinhardtii}

Expression of $C$. reinhardtii GPX5 is strongly induced by the ${ }^{1} \mathrm{O}_{2}$ generated from the photosensitizer RB $[11,13,14]$. To investigate the molecular mechanism by which GPX5 helps maintain ROS homeostasis, we treated the parental strain CC4348, the gpx5 knockout mutant, and the complemented strains L27 or C203 [15] with $2 \mu \mathrm{M}$ RB for $1 \mathrm{~h}$. Then, the total intracellular ROS content was measured, using the fluorescent dye $2^{\prime}, 7^{\prime}$-dichlorofluorescein diacetate (DCFH-DA). Compared to the untreated cells, in CC4348 the level of ROS increased and reached a maximum level (2.1-fold) after $1 \mathrm{~h}$ treatment with RB and then gradually decreased to the initial level after $18 \mathrm{~h}$. In the gpx5 mutant, the level of ROS reached a maximum level (3.3-fold) after $1 \mathrm{~h}$ treatment and then gradually decreased to the initial level after 18 
h. The maximum ROS level in the gpx5 mutant was significantly higher than that in CC4348. In the complemented strain L27, the level of ROS increased to 2.5-fold, which was intermediate between the parental strain and the mutant (Figure 1A). These results demonstrated that GPX5 plays an important role in the removal of ROS.

(A)

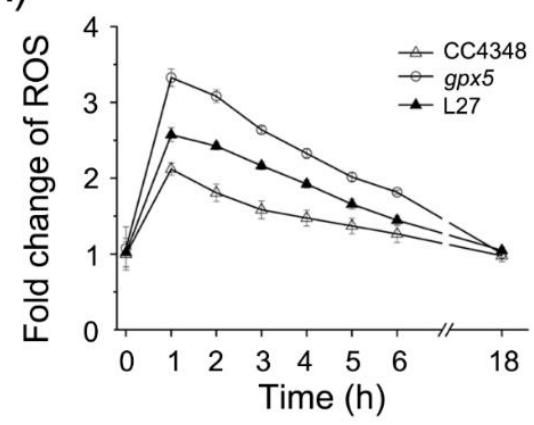

(C)

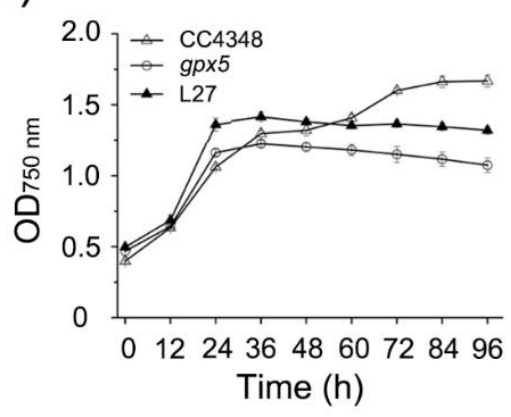

(B)

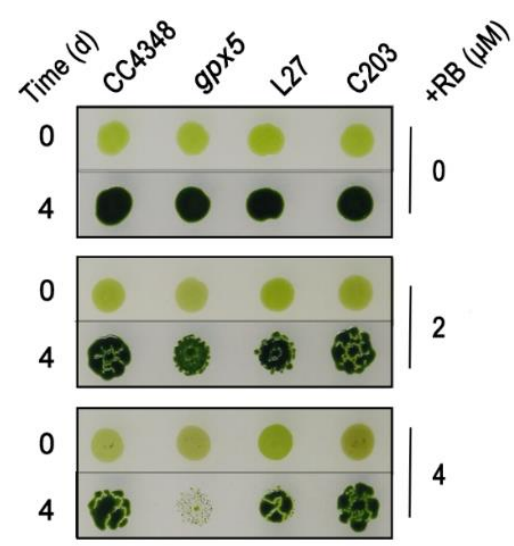

Figure 1. GPX5 maintains ROS homeostasis in Chlamydomonas reinhardtii. (A) Intracellular ROS concentrations in the cells of CC4348, the gpx 5 mutant, and the rescued strain L27 treated with $2 \mu \mathrm{M}$ rose bengal (RB) for $1 \mathrm{~h}$. (B) The effect on the growth of CC4348, the gpx5 mutant, and the complemented lines L27 and C203 treated with 2 or $4 \mu \mathrm{M}$ RB for $1 \mathrm{~h}$. (C) Growth curves of CC4348, the gpx 5 mutant, and L27 cells under laboratory growth conditions. The error bars represent standard deviations of three biological replicates.

To explore the effect of extra ROS on growth, cells of CC4348, the gpx5 mutant, and two complemented strains (L27 and C203) were treated with 2 or $4 \mu \mathrm{M} \mathrm{RB}$ for $1 \mathrm{~h}$. After treatment, the cells were spotted on solid TAP medium, and their growth was monitored after 4 days. As expected, there was no difference in growth among the four strains without RB treatment (Figure 1B). By contrast, for cells that had been treated with 2 or $4 \mu \mathrm{M} \mathrm{RB}$, the spots of the CC4348 strain were much larger and greener after 4 days, compared with the gpx 5 mutant. Indeed, the gpx 5 mutant grew more slowly after being treated with $2 \mu \mathrm{M}$ RB compared to the parental strain and was unable to survive $4 \mu \mathrm{M}$ RB treatment. The two complemented strains, L27 and C203, grew similarly to CC4348. These data indicated that GPX5 plays an important role in maintaining the growth of $C$. reinhardtii cells recovering from oxidative stress.

In order to quantify the effect of GPX5 on growth without RB treatment, we measured the growth rate of CC4348, gpx5 mutant, and L27 cells cultured in TAP complete medium under optimized laboratory growth conditions. CC 4348 cells reached a stable period after $72 \mathrm{~h}$ of incubation, and the $\mathrm{OD}_{750}$ reached 1.6. However, the growth of gpx 5 cells was compromised, and the $\mathrm{OD}_{750}$ was only 1.2 when it reached a stable stage. The growth status of L27 was in between that of CC4348 and gpx5, and the $\mathrm{OD}_{750}$ was 1.4 when it reached a stable stage (Figure 1C). We also found the good correlation between the cell number and $\mathrm{OD}_{750}$ as shown in Supplementary Materials Figure $\mathrm{S1}$. Lower $\mathrm{OD}_{750}$ 
in the stable period of $g p x 5$ mutant suggested the lower cell density. These data indicated that the deficiency of GPX5 effected cell cycle and lowered the population density.

\subsection{The Transcriptome of gpx5 Mutants Under Laboratory Growth Conditions}

To assess the transcriptome of the gpx5 mutant under optimized laboratory growth conditions (no extra oxidative stress added), we performed RNA-seq on CC4348 and gp $x 5$ cells grown at a density of $2 \times 10^{6}$ cells $\mathrm{mL}^{-1}$ in exponential phase in TAP complete medium at $25^{\circ} \mathrm{C}$ under constant illumination of $60 \mu \mathrm{mol} \cdot \mathrm{m}^{-2} \cdot \mathrm{s}^{-1}$ with continuous shaking at $120 \mathrm{rpm}$. The experimental design and analyses are shown in Figure S2. The sequence reads were aligned, and expression level were determined based on the Chlamydomonas reference genome [30,31] (Table S1). Differentially expressed genes (DEGs) were screened with the criteria of false discovery rate (FDR) $\leq 0.05$, fold change $\geq 2$, and at least half of transcript abundance from pairwise comparisons $\geq 10$ reads per $\mathrm{kb}$ of exon per million fragments (RPKM). According to these criteria, we identified 880 DEGs in the gpx5 mutant compared to the parental strain CC4348 under optimized growth conditions, of which 380 DEGs were upregulated and 500 were downregulated (Table S2).

These DEGs were classified by using MapMan [32], employing the Algal Functional Annotation Tool [33] (Figure 2A). We first analyzed transcripts involved in redox metabolism, among which the abundance of 8 transcripts increased, and the abundance of 14 transcripts decreased in the gpx 5 mutant compared to CC4348. The GPX5 transcript was hardly detectable, as expected. GPX4, another member of the GPX family, had a slightly higher transcript abundance in the gpx5 mutant than in CC4348. The other members of GPX family, GPX1-GPX3, had no significant change in transcript abundance in the gpx5 mutant. These data suggested that the expression of other GPX proteins were not upregulated to compensate for the lack of GPX5. 

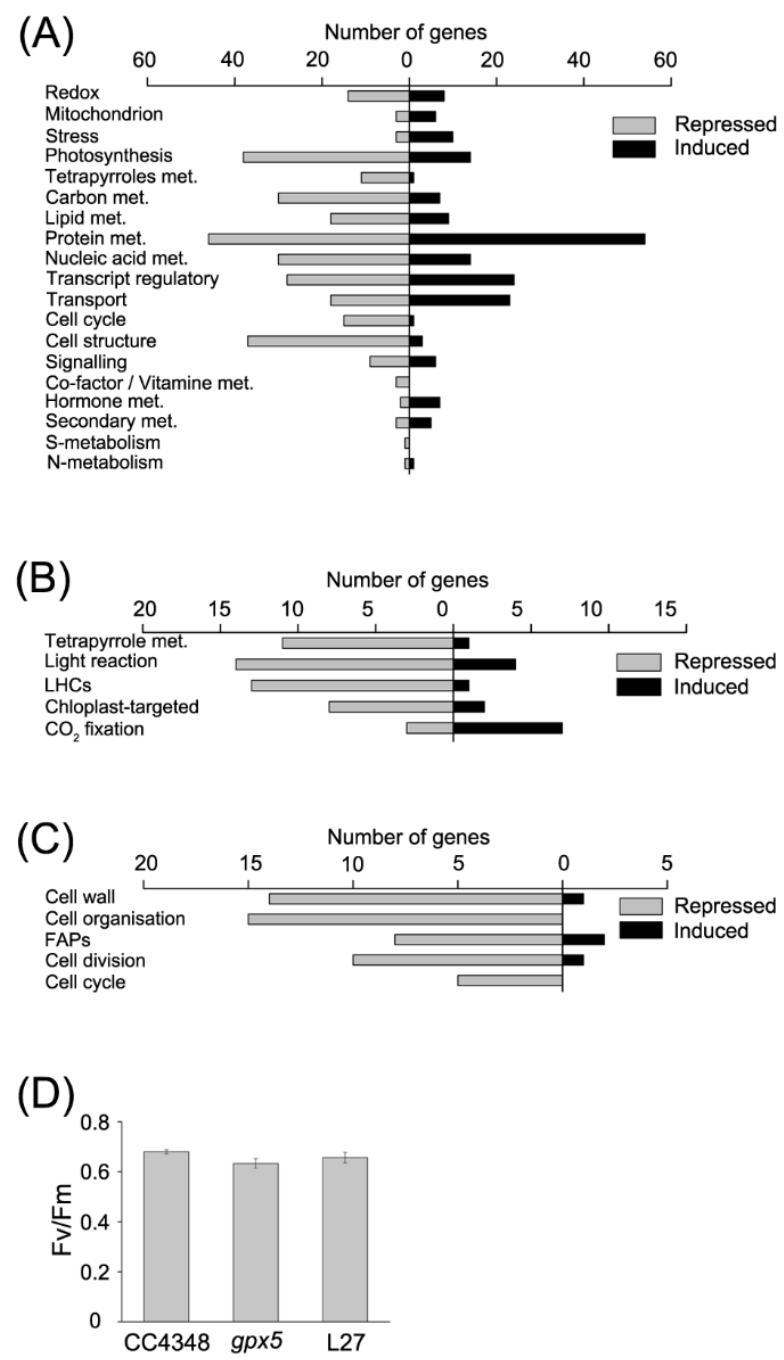

Figure 2. Functional analysis of differentially expressed genes in the gpx5 mutant compared to the parental strain CC4348 under laboratory growth conditions. (A) Functional subcategories of differentially expressed genes (DEGs) in the gpx 5 mutant compared to the parental strain CC4348 under laboratory growth conditions. For clarity, only the genes with assigned functions are shown. Functional analysis of DEGs passing the criteria fold change greater than two (up) or less than 0.5 (down) with FDR $<1 \%$, based on the MapMan functional classes; met, metabolism. (B) List of the DEGs related to photosynthesis and tetrapyrrole metabolism in the gpx5 mutant compared to the parent strain CC4348 under laboratory growth conditions. LHC, light-harvesting complex. (C) List of the DEGs related to cell structure and the cell cycle in the $g p x 5$ mutant compared to the parental strain CC 4348 under laboratory growth conditions. FAPs, flagellar-associated proteins. (D) The maximum efficiency of PSII (Fv/Fm) of CC4348, the gpx 5 mutant, and L27 cells at a density of $2 \times 10^{6}$ cells mL $^{-1}$ in exponential phase under laboratory growth conditions. The error bars in the graph represent standard deviations of three biological replicates.

Among the eight upregulated transcripts related to redox in the gpx5 mutant under optimized growth conditions, the transcripts of two nucleoredoxins (NRX2 and NRX3) and one sulfiredoxin (SRX1) increased by more than 4.5-fold. As antioxidants, NRXs reduce other proteins by cysteine thiol-disulfide exchange, functioning as protein disulfide reductases (PDIs). Similarly, PDI5 transcript abundance significantly increased more than two-fold in the gpx5 mutant. NRX2, NRX3, SRX1, and PDI5 can reduce the disulfide bond of oxidized proteins, attenuating the effects of oxidative stress. The abundance of transcripts of VTC2 (Cre13.g588150), encoding GDP-L-galactose phosphorylase, a key enzyme in de novo ascorbate synthesis [34], increased by about three-fold in the gpx5 mutant, 
suggesting that the synthesis of the antioxidant ascorbate (ASC) was induced. These data suggested that some mechanisms of ROS scavenging were enhanced in the gpx5 mutant, and that, even under unstressed mixotrophic conditions, the presence of ROS occurred and was sufficient for cell signaling.

In order to detect the effects of GPX5 deficiency on basal metabolism, we found the modifications in the CCM occurred in the mutant. Most of genes encoding proteins involving in CCM were upregulated in the gpx 5 mutant, including some low- $\mathrm{CO}_{2}$-inducible proteins (CCP2, LCI24, LCI5, LCIB, and LCIE). Moreover, we also analyzed the expression change of genes in the chlororespiraton. Among them, seven out of nine transcripts showed no difference in abundance between CC4348 and the gpx5 mutant, including glycolate dehydrogenase (GYD1, Cre06.g288700), phosphoglycolate phosphatase (PGP1 and PGP2 encoding by Cre03.g168700 and Cre10.g438100, respectively), serine hydroxymethyltransferase (SHMT1, Cre16.g664550), alanine-glyoxylate transaminase (AGT1 and AGT2), and serine glyoxylate aminotransferase (SGA1). Only hydroxypyruvate reductase (HPR1) and alanine aminotransferase (AAT1) were downregulated in the gpx5 mutant. These results meant the deficiency of GPX5 had few effects on chlororespiraton.

Transcript abundances of the glutathione S-transferase (GST) genes (GST1 and GST10) were very low in the gpx 5 mutant (4 and 6 RPKM, respectively) compared to that of either GST1 or GST10 in CC4348. GSTs detoxify endogenous or xenobiotic compounds and prevent oxidative damage to cells [35-37]. Stress-inducible GSTs with glutathione peroxidase activity can protect plants from oxidative injury [38]. Moreover, GSTS are biomarkers of the response to stress and overexpression of GSTs enhances tolerance to abiotic stress $[39,40]$. The transcript abundance of GSTs in the gpx5 mutant was not higher than that in CC4348. This suggests that the gpx5 mutant was not experiencing oxidative stress when grown on TAP complete medium under our optimized laboratory growth conditions.

Mutation of GPX5 resulted in the adjustment of metabolic processes. The transcripts of 38 photosynthesis-related genes were decreased and 14 photosynthesis-related transcripts were increased in the gpx5 mutant. Most of the transcripts encoding antenna proteins showed a downward trend, except the LHCA3 transcript, encoding a chlorophyll a/b binding protein of light-harvesting complex (LHC) I, which increased about 2-fold in abundance in the gpx5 mutant. Moreover, nearly all of the genes involved in tetrapyrrole synthesis were downregulated, except for UPS1, which encodes uroporphyrinogen-III synthase (Cre09.g409100) (Figure 2A,B). To confirm the effect of GPX5 deficiency on photosynthesis, we measured the maximum efficiency of photosystem II (PSII) (Figure 2D). The results showed a slightly reduction in photosynthetic efficiency of the gpx5 mutant compared to CC4348, suggesting that the gpx5 mutant might minimize ROS accumulation by slowing down photosynthesis, which is a major source of ROS.

Among 37 DEGs encoding proteins related to carbon metabolism, including glycolysis, gluconeogenesis, starch synthesis, starch degradation, and the TCA cycle, 30 transcripts were downregulated in the gpx 5 mutant. The transcript with the largest decrease in level encodes malate synthase (MAS1), which uses acetyl coenzyme A to synthesize malic acid. The abundance of the MAS1 transcript decreased from 756 RPKM in CC4348 to 221 RPKM in the gpx5 mutant. We also detected the isocitrate lyase (ICL1) transcript, which decreased in abundance from 785RPKM in CC4348 to 282 RPKM in the gpx5 mutant under laboratory growth conditions; and so did the transcripts of citrate synthase (CIS2) and acetyl-CoA syntheses/ligase (ACS3). The transcript of CIS2 and ACS3 decreased from 80 to 62 RPKM, and from 2508 to 1361 RPKM, respectively. These data would simply suggest that, since gpx5 is growing more slowly than the control, the transcripts encoding transcripts involved in the glyoxylate cycle are in lower amounts. Almost all genes encoding enzymes in the glycolysis/gluconeogenesis pathway were downregulated in the gpx5 mutant, except fructose-1,6-bisphosphate aldolase (FBA1). Nevertheless, the reaction from fructose-1,6-diphosphate to glyceraldehyde-3-phosphate catalyzed by FBA1 is reversible, so it might not affect the overall metabolic slowdown of the glycolysis/gluconeogenesis pathway.

Lack of GPX5 also affected lipid metabolism, resulting in 27 lipid-related DEGs (9 upregulated and 18 downregulated). The transcript abundance of KAS2 and KAS3, which encode $\beta$ 
ketoacyl acyl-carrier-protein synthase II and synthase III, respectively, and HAD1, encoding 3-hydroxyacyl-carrier-protein dehydratase, decreased to 0.07 - to 0.35 -fold in the gpx5 mutant. The transcripts of $F A D 6$, encoding chloroplast omega-6-fatty acid desaturase, increased in abundance by 2.3 -fold, reaching 1374 RPKM. Increasing the unsaturation of membrane lipids in cyanobacteria can improve the tolerance to low temperature [41]. Therefore, upregulation of the expression of genes encoding fatty acid desaturases may affect the metabolism of unsaturated fatty acid in the gpx 5 mutant.

Functional classification showed that 15 out of 18 DEGs related to amino acid metabolism were downregulated in the gpx5 mutant. The abundance of transcripts encoding the enzymes involved in arginine synthesis were significantly decreased, including arginosuccinate synthase (Cre09.g416050, AGS1), acetylglutamate kinase (Cre01.g015000, AGK1), argininosuccinate lyase (Cre01.g021251, ARG7), N-acetylglutamate synthase (Cre16.g694850, NGS1), and N-acetyl-gamma-glutamyl-phosphate reductase (Cre03.g146187). These observations suggested that decreasing synthesis of arginine might play some function in response to GPX5 deficiency or the resulting ROS imbalance.

Besides the effect of oxidative stress on metabolism, transcripts related to the cell cycle and structural proteins were significantly downregulated in the gpx5 mutant (Figure 2A,C). These effect on cell-cycle-related genes is consistent with the effect on growth described above (Figure 1C). In our previous study [15], we found that the gpx5 mutation died earlier than the parental stain CC4348 when cultured in nitrogen-free medium. The grow difference between the mutant gpx5 and parental strain suggested that the deficiency of GPX5 in the mutant not only affected ROS homeostasis, but also affected cell growth.

We also identified DEGs related to nucleotide or nucleic acid metabolism, transcript regulatory, mitochondrial electron transport chain (mETC) or encoding transporters. For example, the transcripts encoding a bZIP transcription factor BLZ8 (Cre12.g501600) and a zinc finger transcription factor (Cre03.g156250) increased about 2.5-fold, with transcript abundance increasing from 108 and 105 RPKM in CC4348 to 258 and 253 RPKM in the gpx 5 mutant, respectively.

In summary, intracellular antioxidant metabolism and transport appeared to adjust to maintain redox homeostasis in the gpx5 mutant. The expression of genes related to carbon metabolism, lipid metabolism, and synthesis of chlorophyll were depressed, and the efficiency of photosynthesis decreased in the gp $x 5$ mutant rather than in CC4348. These overall multifaceted changes in transcription slowed the growth of the gpx5 mutant cells.

\subsection{Transcriptome Responses to ${ }^{1} \mathrm{O}_{2}$ in Parental Strain CC4348 Cells}

To address the role of GPX5 in response to oxidative stress, we first studied the mechanisms by which parental strain $C$. reinhardtii cells respond to oxidative stress. To this end, we performed RNA-seq on CC4348 cells at 0,30 , and 60 min after the addition of $1 \mu \mathrm{M}$ RB to the medium. Using the same criteria as before, we identified 708 DEGs after the 30-min RB treatment, accounting for $4.7 \%$ of the total genome. These DEGs included 322 upregulated transcripts and 386 downregulated transcripts (Table S3).

ROS homeostasis was disturbed in CC4348 cells under oxidative stress. The transcripts of 22 redox metabolism-related genes were upregulated, and six were downregulated (Figure $3 \mathrm{~A}$ ). The transcript abundances of genes encoding ROS-scavenging enzymes, such as ascorbate peroxidase (APX1), glutathione reductase (GSR1), GPX5, and the GST family, were upregulated after ${ }^{1} \mathrm{O}_{2}$ stress. GPX5 has been reported to be highly induced by ${ }^{1} \mathrm{O}_{2}[11,12]$, and the transcript abundance of GPX5 increased 13.5-fold after treatment with RB for $30 \mathrm{~min}$ in CC4348 cells (Table S3). Another non-selenocysteine GPX gene (GPX4) and selenium-dependent GPX gene (GPX2) showed no difference in transcript abundance. Additionally, the transcript of GPX3 was barely detectable. Interestingly, one selenium-dependent GPX gene (GPX1) showed the opposite expression pattern, as it was downregulated from 100 to 6 RPKM after 30 min of RB treatment. The active site of GPX1 is selenocysteine, which is easily oxidized and has strong reducibility. Generally, antioxidants function actively during oxidative stress, so the 
downregulation of GPX1 was unexpected. One possible explanation is that different GPX genes are induced by different oxidative stresses.
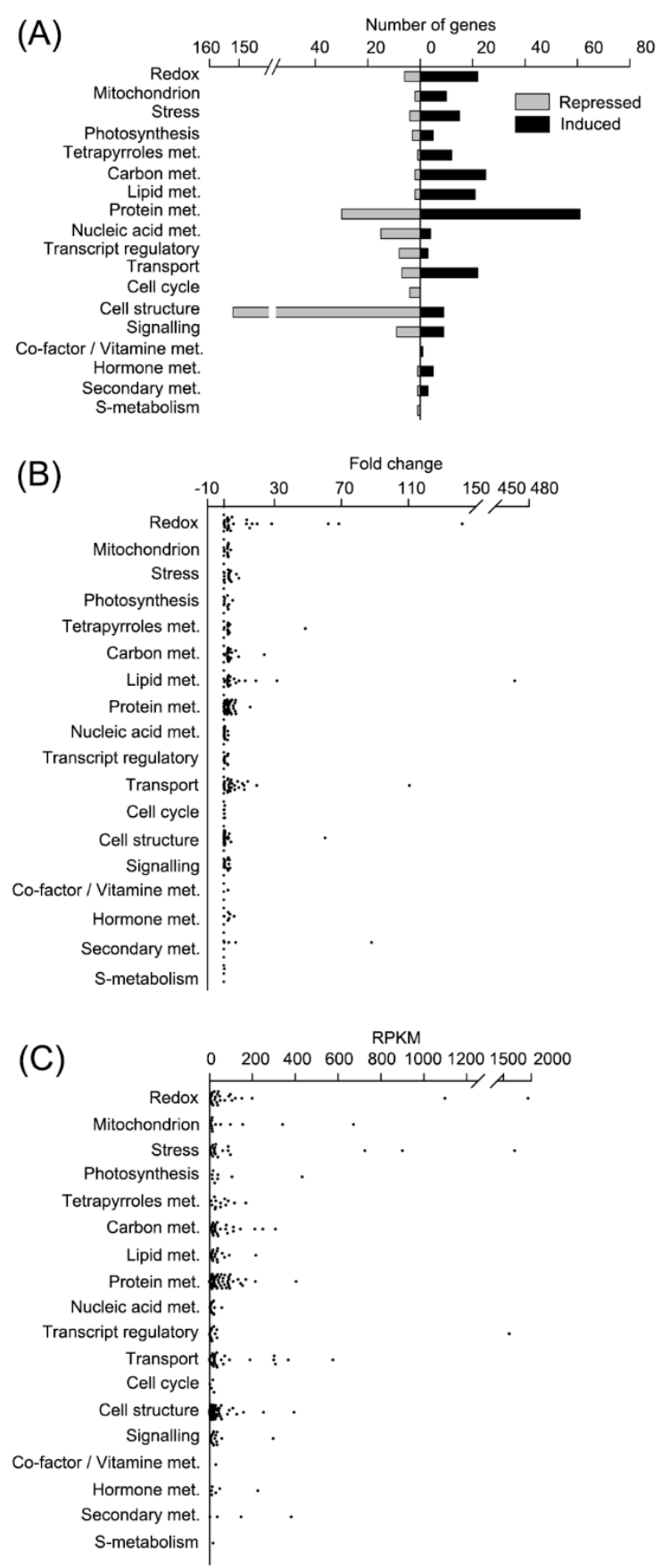

Figure 3. Functional analysis of differentially expressed genes in CC4348 after being treated with $1 \mu \mathrm{M}$ RB for $30 \mathrm{~min}$. (A) Functional subcategory of differentially expressed genes (DEGs) in CC4348 treated with $1 \mu \mathrm{M} \mathrm{RB}$ for $30 \mathrm{~min}$. For clarity, only the genes with assigned functions are shown. Functional analysis of DEGs passing the criteria of fold change greater than two (up) or smaller than 0.5 (down) with FDR $<1 \%$, based on the MapMan functional classes; met, metabolism. (B) The fold change of transcript abundance of DEGs in (A). The X-axis indicated the logarithm based two of the ratio of transcript abundance of genes after treated with $1 \mu \mathrm{M}$ RB for 30 min to control in CC4348. (C) The relative transcript abundance of DEGs in (A). The X-axis indicated the value in unit of RPKM of transcript abundance after treated with $1 \mu \mathrm{M}$ RB for $30 \mathrm{~min}$ in CC4348. 
We noted that most genes involved in redox metabolism were upregulated in CC4348 cells after treatment with $1 \mu \mathrm{M}$ RB for $30 \mathrm{~min}$. Among the 28 redox-related DEGs, the transcripts of ferritin2, encoding a ferritin-like protein (Cre01.g033300) had the greatest fold change, from 14 to 1944 RPKM, an increase of about 140-fold (Figure 3B and Table S3). Ranked second were SOUL heme-binding protein genes (SOUL1 and SOUL2), whose transcripts increased more than 60 -fold. This indicated that heme might play an important role in maintaining redox homeostasis. Moreover, GST family genes also responded to RB treatment, including Cre07.g342100, GST1 (Cre16.g688550), GST10 (Cre12.g559800), and PDO2 (Cre12.g559800) which belong to the GST KAPPA class. Among them, Cre07.g342100 encodes a GST, whose transcript increased sharply by 29 -fold within $30 \mathrm{~min}$ after ${ }^{1} \mathrm{O}_{2}$ stress, reaching a peak of 121 RPKM. Thioredoxin superfamily proteins and proteins containing thioredoxin domains, including NRX2, protein disulfide-isomerase A6 (PDIA6, Cre07.g326600), and r53.5-related protein LCI7 (Cre06.g263550) also responded to ${ }^{1} \mathrm{O}_{2}$. Among them, PDIA6 and LCI7 were upregulated more than 15-fold within 30 min of the treatment.

To confirm the RNA-seq data, we performed qRT-PCR on a set of DEGs, including the GPXs, APX1, Mn superoxide dismutase (MSD2), SOUL1, SOUL2, LCI7, and the GSTs (Figure 4). The expression pattern from qRT-PCR was similar to that from RNA-seq, and the coefficient of association between them was about 0.84 (Figure S3). Both RNA-seq data and qRT-PCR results showed that most of the transcript abundances of enzymes to detoxify ROS were upregulated. Overall, after the RB treatment, the antioxidant system was activated in CC4348 cells via the expression of related genes, providing sufficient antioxidant proteins to eliminate the ${ }^{1} \mathrm{O}_{2}$ stress generated by $\mathrm{RB}$.
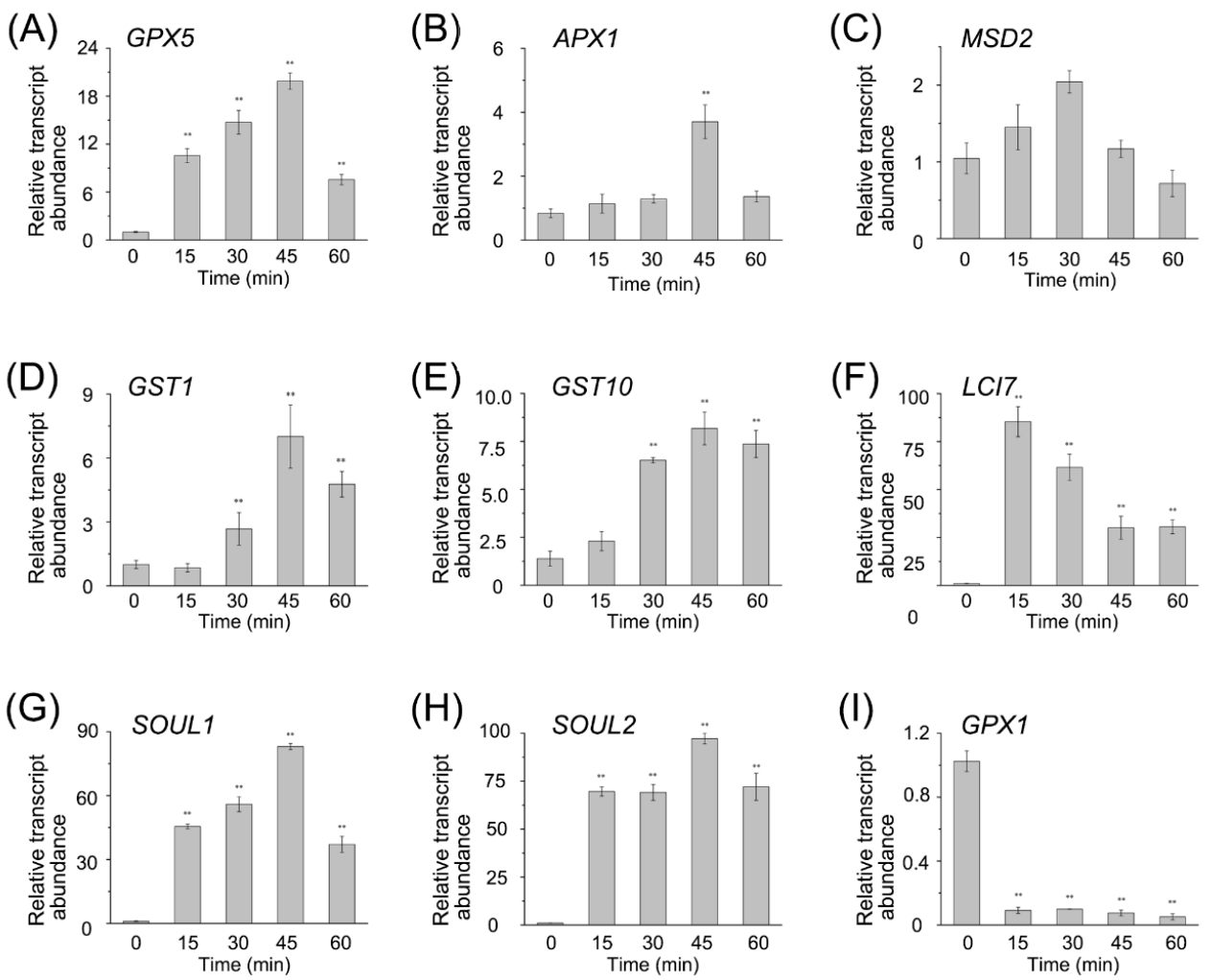

Figure 4. Transcriptional changes of antioxidant-related genes in RB-treated CC4348 cells. The transcript abundance of GPX5 (A), APX1 (B), MSD2 (C), GST1 (D), GST10 (E), LCI7 (F), SOUL1 (G), and SOUL2 $(\mathbf{H})$ and GPX1 (I) at 15, 30, 45, and 60 min after treatment with $1 \mu \mathrm{M}$ RB in CC4348 were detected, using qRT-PCR. C $\beta L P$ was used as an internal control to normalize the data. The error bars indicate standard deviation of biological triplicates. The asterisks represent statistical significance between every treated group and the control, which was determined by $t$-test $(n=3, P<0.01)$. 
RNA-seq data also show that treatment with RB induced the expression of an iron-assimilating protein gene (FEA1, Cre12.g546550) and stress-related molecular chaperones, which assist in proper protein folding. The transcript abundance of $F E A 1$ increased to 1703 RPKM after $30 \mathrm{~min}$ of stress from an initial value of 590 RPKM. Molecular chaperones also responded strongly. For example, the transcript abundance of HSP70A increased about 3-fold, from 261 to 726 RPKM, while HSP90A increased more than 3-fold, from 275 to 901 RPKM after treatment with RB for 30 min, respectively (Figure 3C and Table S3). Genes encoding heat shock proteins (HSP22E, HSP22F, HSP70C, HSP70E, HSP90B, and HSP90C), CLPB3 (a ClpB chaperone belong to HSP100 family, Cre02.g090850), HOP1 (HSP70-HSP90 organizing protein), CPN60C (Chaperonin 60C), a DnaJ-like protein DNJ34, and HSP90-cochaperone (Cre07.g341550) were also all upregulated in CC4348 cells after ${ }^{1} \mathrm{O}_{2}$ stress for $30 \mathrm{~min}$. This upregulation of the expression of molecular chaperones could be due to the accumulation of incorrectly folded or oxidatively damaged proteins due to oxidative stress. In support of this conclusion, we found that the transcript abundance of 17 proteasome-related genes, encode proteins involved in ubiquitination-dependent proteasome-degradation pathways, including ubiquitin-activated enzyme E1 (UBA1), ubiquitin ligase E3 (Cre12.g533750, Cre12.g500550, Cre12.g501450, and Cre11.g476250), and seven proteasome subunits, increased more than 2-fold. In addition, the transcripts of genes encoding seven proteases or metalloproteinases were upregulated.

We found 27 DEGs related to carbon metabolism, including glycolysis, the TCA cycle, and oxidative pentose phosphate pathway (OPP), and expression of 25 genes was upregulated after treatment with RB for 30 min. Four rate-limiting enzymes, a fructose-bisphosphatase (FBP1), phosphofructokinases (PFK1 and PFK2), and a pyruvate kinase (PYK4) involved in glycolysis were all induced. Notably, the PYK4 transcript increased about 5-fold to peak at 112 RPKM. Transcripts encoding another three key enzymes involved in the TCA cycle, 2-oxoglutarate dehydrogenase, citrate synthase, and isocitrate dehydrogenase, were also upregulated more than 2.5-fold. Only carbonic anhydrase (CAH8) and chitinase (Cre07.g317250) showed a different expression pattern relative to other proteins involved in carbon metabolism. On the whole, glycolysis and the TCA cycle pathway showed a rising trend, which might provide more ATP to respond to oxidative stress.

The expression of 21 transcripts related to lipid metabolism was upregulated, and only two genes were downregulated. Transcripts encoding cyclopropane fatty acid synthase (CFA1 and CFA2) increased in abundance by about 19-fold and 32-fold, to 217 and 70 RPKM, respectively (Figure 3B,C, Table S3). The transcript of a sterol-sensing 5-transmembrane protein (SSD1) could hardly be detected under normal growth conditions, but after $30 \mathrm{~min}$ of RB treatment, the SSD1 transcript reached 10 RPKM. SSD1 can function as a lipid transporter; therefore, the increase of SSD1 transcript implied that lipid composition changed in response to oxidative stress.

Enriched classes of DEGs also included transcripts related to hormone metabolism. For example, seven enzymes involved in jasmonic acid (JA) biosynthesis were induced after RB treatment for 30 min, including Acyl-CoA oxidase (ACO2, ACO3, and ACO4) and 3-hydroxyacyl-CoA dehydrogenase (HCD1), acyl-coenzyme A thioesterase 9 (TEH6, Cre16.g683350), lnoleate 13S-lipoxygenase (13-LOX, Cre12.g512300). Notably, OPR encoding 12-oxophytodienoic acid reductase (Cre03.g210513) increased in transcript abundance by about 6-fold, from 38 to 226 RPKM. The identity between 13S-lipoxygenase (13-LOX, Cre12.g512300) in C. reinhardtii to lipoxygenase (LOX1, AT1G55020) in Arabidopsis thaliana, 12-oxophytodienoic acid reductase (OPR, Cre03.g210513) in C. reinhardtii to oxophytodienoate-reductase 3 (OPR3, AT2G06050) in Arabidopsis thaliana is 36\% and 46\%, respectively, so we are convinced to conclude that jasmonic acid biosynthesis occurred in C. reinhardtii. JA regulates the induced defense responses to heavy metals in the green alga Chlorella vulgaris [42] and cold tolerance in Arabidopsis leaves [43]. Therefore, we speculated that JA might also play an important role in responding to RB treatment in C. reinhardtii.

In response to RB treatment, $94 \%$ of the genes encoding cell-structure-related proteins were downregulated. Moreover, 88\% (134 DEGs) of the downregulated genes encoded flagellar associated proteins (FAPs), including Intraflagellar Transport (IFT) proteins. To further explore whether oxidative stress affected the expression of FAPs, we examined CC125 cells treated with $1 \mu \mathrm{M}$ RB for $1 \mathrm{~h}$. Because CC4348 strain is the mutant without flagella, widely used CC125 strain was used to measure 
the length and ratio of flagella. Up to $37 \%$ of these cells lost their flagella at $12 \mathrm{~h}$. The length of flagella did not change (Figure S4), suggesting that RB induced deflagellation, not resorption.

Overall, ${ }^{1} \mathrm{O}_{2}$ activated the detoxication of ROS, stimulated increases in the abundance of HSP family proteins and ubiquitin-mediated proteolysis, upregulated carbon metabolism and lipid metabolism, and induced deflagellation.

\subsection{Transcriptome Responses to ${ }^{1} \mathrm{O}_{2}$ in the gpx 5 Mutant}

To address the function of GPX5 in response to oxidative stress, we compared the transcriptomes of the parental strain $\mathrm{CC} 4348$ and the gpx 5 mutant during ${ }^{1} \mathrm{O}_{2}$ stress. RNA-seq was performed on the parental strain CC4348, the gpx5 mutant, and complemented strain L27 at 0,30, and 60 min after the addition of $1 \mu \mathrm{M}$ RB (Figure 5). Using the same screening criteria, we identified 1852 DEGs in CC4348 or the gpx 5 mutant under oxidative stress. To compare the responses of CC4348 and the gpx5 mutant, we divided the 1852 DEGs into three categories. Group A were upregulated during the oxidative stress in CC4348 and included 947 genes. Group B did not have significantly different expression during the treatment and had 478 genes. Group $\mathrm{C}$ were downregulated during the response to ${ }^{1} \mathrm{O}_{2}$ in $\mathrm{CC} 4348$. An overview of the functions related to these transcript categories is presented in Table 1, with an emphasis on differences between CC4348 and gpx5 mutant cells (Table S4).

(A)

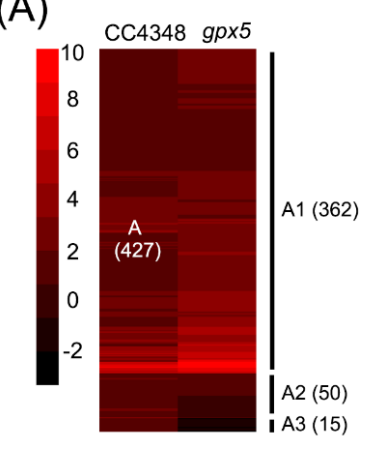

(B)

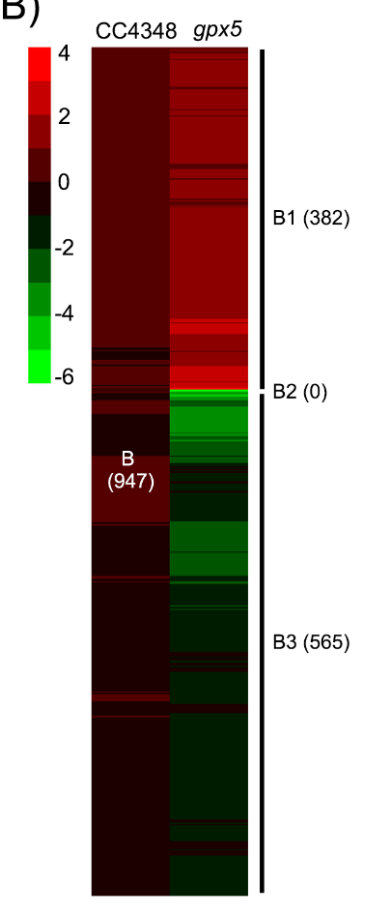

(C)

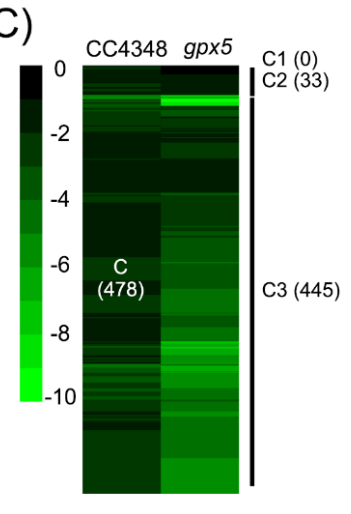

Figure 5. Comparison of the transcriptional response of CC4348 versus the gpx5 mutant after treatment with $1 \mu \mathrm{M}$ RB for $30 \mathrm{~min}$. The cluster diagrams depict the fold change in transcript levels determined by RNA-seq. The $\log _{2}$ relative expression values of 1 and -1 were selected as thresholds to designate the categories of transcripts that accumulated and declined, respectively. (A) The upregulated DEGs in CC4348 were categorized into A1 (upregulated in the gpx5 mutant), A2 (no significant change in the gpx5 mutant), and A3 (down regulated in the gpx5 mutant). (B) The genes without significant changes in CC4348 but with significant changes in the gpx5 mutant were categorized into B1 (upregulated in the gpx 5 mutant), B2 (no significant change in the gpx5 mutant), and B3 (downregulated in the gpx5 mutant). (C) The downregulated DEGs in CC4348 were categorized into C1 (upregulated in the gpx5 mutant), C2 (no significant change in the gpx 5 mutant), and C3 (downregulated in the gpx5 mutant). 
Table 1. Overview of metabolic pathways and cellular processes in each subgroup from Figure 5. The numbers in brackets represent the counts of according subgroups.

\begin{tabular}{|c|c|c|}
\hline $\begin{array}{c}\text { Specific in (CC4348 + RB) } \\
\text { Low ROS }\end{array}$ & Shared & $\begin{array}{c}\text { Specific in }(g p x 5+\mathrm{RB}) \\
\text { High ROS }\end{array}$ \\
\hline $\begin{array}{c}\text { Induced } \\
\text { A2+A3 }(50+15) \\
\text { Chlorophyll metabolism, } \\
\text { Transport ATPases, } \\
\text { Transcript regulatory, } \\
\text { FEA1. }\end{array}$ & $\begin{array}{c}\text { A1 (362) } \\
\text { a ROS detoxification, } \\
\text { Ubiquitin-mediated proteolysis, } \\
\text { Glycolysis, OPP, } \\
\text { Ferritin2. }\end{array}$ & $\begin{array}{c}\text { B1 (382) } \\
\text { Mitochondrial electron transport, } \\
\text { TCA cycle, } \\
\text { b Ubiquitin-mediated proteolysis, } \\
\text { Secretory pathway. }\end{array}$ \\
\hline $\begin{array}{c}\text { Repressed } \\
\text { C2 (33) } \\
\text { Nucleotide metabolism, } \\
\text { Transcript regulatory. }\end{array}$ & $\begin{array}{c}\text { C3 (445) } \\
\text { c Cell structure proteins, } \\
\text { Selenoproteins. }\end{array}$ & $\begin{array}{l}\text { B3 (565) } \\
\text { Signal transduction, } \\
\text { Iron transporters. }\end{array}$ \\
\hline
\end{tabular}

( ${ }^{a}$ Some transcripts were also found in class B1. ${ }^{\mathrm{b}}$ Some transcripts were also found in class A1. ${ }^{\mathrm{c}}$ Some transcripts were also found in class B3. FEA1, Fe-assimilating protein; Ferritin2, ferritin-like protein.)

The group A DEGs were further classified into three subgroups, A1 (362 genes, upregulated in CC4348 and gpx5), A2 (50 genes, upregulated in CC4348, not changed in gpx5), and A3 (15 genes, upregulated in CC4348 and downregulated in $g p x 5$ ) (Figure 5A). The highest enriched categories of the subgroup A1 proteins include ROS detoxification, carbon metabolism, and ubiquitin-dependent proteolysis. In subgroup A1, 31 transcripts encoded proteins regulating redox homeostasis, including APX1, GSR1, GST6, GST10, VTC2, TRXs, and SRX1. These antioxidases or antioxidant proteins responded to oxidative stress independent of GPX5.

Although the transcript abundance of most genes related to carbon metabolism was lower in the gpx 5 mutant than that in CC4348 under normal growth conditions, transcripts encoding proteins related to glycolysis and the oxidative pentose phosphate pathway (OPP), such as FBP1, PFK1, PFK2, and transaldolases (TAL1, TAL2) from subgroup A1, were upregulated in gp $x 5$ in the same pattern as in CC4348 after RB treatment. In particular, the TAL1 transcript increased from 26 to 194 RPKM in gpx5 and from 89 to 250 RPKM in CC4348. Increasing glycolysis metabolism and OPP would likely produce more NADPH to counteract the oxidative environment in cells.

Genes from subgroups A2 and A3 (upregulated in CC4348 and unchanged or downregulated in gpx5) are mainly involved in chlorophyll metabolism or related to redox or transport. Eight transcripts encoding proteins involved in chlorophyll synthesis from subgroup A2 were significantly increased in abundance in CC4348, whereas those mostly remained constant in the gpx 5 mutant after RB treatment. Magnesium chelatase subunit transcripts (CHLD, CHLI1, and CHLI2) were upregulated by more than 2-fold (peak more than 50 RPKM) in CC4348 after treatment, but did not significantly change in the gpx 5 mutant. Subgroup A3 had only one transcript related to chlorophyll synthesis, CPX1 (coproporphyrinogen III oxidase), which was upregulated in CC4348 but downregulated in the gpx5 mutant. These observations suggested that the chlorophyll content would increase in CC4348 but not in gpx5 after RB treatment.

Five transcripts from subgroup A2 and 3 transcripts from subgroup A3 encoded putative transporters, including transporters in vesicular trafficking (Cre04.g224800 and Cre09.g394954), mitochondrial oxoglutarate/malate carrier protein (Cre11.g467535), sodium-exporting ATPase (Cre17.g744447), and major facilitator superfamily transporter (MFT10, Cre02.g095076) from subgroup A2, and sodium/bile acid cotransporter 7 (Cre02.g095086), MFT26 (Cre12.g512200), and a solute carrier family 35 member (Cre06.g286200) from A3.

We also observed that the abundance of transcripts encoding thioredoxin peroxidase (Cre16.g654250) from subgroup A2 and NRX2 from subgroup A3 increased in CC4348 under oxidative stress, but not in the gp $x 5$ mutant. The transcript for a $60-\mathrm{kDa}$ SS-A/Ro ribonucleoprotein (Cre17.g725750) from subgroup A3 increased in abundance, from 35 to 93 RPKM in CC 4348 after RB treatment for 30 min, 
but decreased from 136 to 37 RPKM in the gpx 5 under the same conditions. This RNA-binding protein is differentially expressed depending on GPX5 under oxidative stress, suggesting that it regulates the downstream response.

Genes in group B were similarly classified into subgroups, according to their response in the gpx5 mutant under oxidative stress. Among them, 382 genes, which were designated as B1, were upregulated in the gpx5 mutant, and 565 genes, designated B3, were downregulated in the gpx5 mutant (Figure 5B). Therefore, genes in subgroup B1 were specifically upregulated in the gp 55 mutant, and genes in subgroup B3 were specifically downregulated in the gpx5 mutant.

Unlike the parental strain CC4348, the gpx 5 cells had high concentrations of ROS after RB treatment (Figure 1A). The high concentration of ROS induced transcripts from subgroup B1, which are mainly associated with the METC, carbon metabolism, protein metabolism, and endosomal sorting complex required for transport (ESCRT). The transcript abundance of genes encoding cytochrome c oxidase subunits (COX2A and COX3), cytochrome c oxidase assembly factor (SCO1), ubiquinone cytochrome c oxidoreductase subunit (QCR1), and eight mitochondrial ATP synthase subunits in the mETC remained stable in CC4348, but increased by 2- to 5-fold in the gpx5 mutant after oxidative stress treatment. Besides, we found a type II NAD(P)H dehydrogenase (NDA1), an alternative oxidase (AOX1), complex IV (SCO1 and PET191) upregulated, and this fits well the data published by Sabeeha S. Merchant [44]. They showed transcripts encoding proteins of the most mETC genes not changed and the $\mathrm{O}_{2}$ consumption decreased. These results suggested that ATP produced by respiration is in smaller amounts in stress conditions.

Transcripts encoding proteins involved in carbon metabolism, especially the TCA cycle and starch metabolism, were also enriched in subgroup B1. Indeed, 12 transcripts encoding proteins involved in the TCA, phosphoenolpyruvate carboxykinase (PCK1), mitochondrial pyruvate dehydrogenase complex alpha subunit (PDC1), isocitrate dehydrogenase (IDH1 and IDH2), Dihydrolipoamide succinyltransferase (OGD2), succinyl-CoA ligase (SCL1 and SCL2), succinate dehydrogenase (SDH1 and SDH3), fumarase (FUM1), and NAD-dependent malate dehydrogenase (MDH3 and MDH4) were significantly increased only in the gpx5 mutant after RB treatment for $30 \mathrm{~min}$. In addition, OGD1, CIS1, and IDH3 increased in the gpx 5 mutant and CC4348. The upregulation of the TCA cycle, along with increased mETC activity, likely supply more energy to respond to oxidative stress in the gpx5 mutant.

A comparison of $g p x 5$ and CC 4348 also showed that transcripts related to ubiquitin-mediated proteolysis were induced after oxidative stress both in CC4348 and gpx5 cells, including 30 transcripts from subgroup A1 and 33 transcripts from subgroup B1. Almost all transcripts related to the ubiquitin-proteasome system increased in abundance during the 60 -min oxidative stress treatment in CC4348 (Figure S5 and Table S5). Under the control conditions (0 min), the abundance of these transcripts was lower in the gpx5 mutant than that in CC4348. After $60 \mathrm{~min}$ of RB treatment, these transcripts showed a dramatic upregulation in the $g p x 5$ mutant, significantly larger than that of CC4348. Before the treatment $(0 \mathrm{~min})$, the abundances of these transcripts were similar in CC4348 and gpx5, but they increased significantly after addition of RB in L27, and the increase was between that of CC4348 and gpx 5 cells. This increase suggested that ROS stimulated the upregulation of transcripts encoding proteasome-related subunits. As ROS concentration increased, protein stress increased, resulting in upregulation of the ubiquitin-proteasome system.

Oxidative stress also induced 11 transcripts encoding proteins in the secretory pathway or vacuolar assembly/sorting proteins (VPS2A, VPS26, VPS46, and VPS60) from subgroup B1. These transcripts increased in abundance specially in the gpx 5 mutant after RB treatment. These data suggest that high ROS levels accelerated the transport and exchange of metabolites between cells.

Transcripts from subgroup B3 genes were specifically downregulated in the gpx5 mutant, including ten adenylate cyclase or guanylate cyclase subunit transcripts and five $3^{\prime}, 5^{\prime}$-cyclic nucleotide phosphodiesterase transcripts, whereas there was no significant difference in CC4348. Adenylate cyclase and guanylate cyclase catalyze the formation of cAMP and cGMP, respectively. As secondary messengers, cAMP or cGMP activate kinases. The high concentration of ROS could inhibit some cAMP or 
cGMP signaling pathways. In addition, subgroup B3 included transcripts encoding Mitogen-Activated Protein Kinase (MAPK) signaling pathway factors. For example, MAPKKK7 (Mitogen-Activated Protein Kinase Kinase Kinase) was specifically downregulated, from 19 to 9 RPKM in the gpx5 mutant, but its expression remained stable at about 16 RPKM in CC4348. ROS also affected the calcium signal as mediated by calmodulin. Transcripts encoding calmodulins (Cre03.g210177 and Cre03.g170200), a Ca ${ }^{2+}$-transporting ATPase (Cre12.g505350), and a calcium-binding protein (Cre16.g680500) from subgroup B3 were specially downregulated in the gpx5 mutant but showed no significant difference in CC4348. This suggests that signal transduction including cAMP or cGMP, MAPK pathways, and $\mathrm{Ca}^{2+}$ signaling were inhibited in response to high concentrations of ROS.

Two iron transporter genes, IRT2, encoding an iron-responsive ZIP family transporter (Cre12.g530350) and FTR1, encoding an iron transporter (Cre03.g192050), responded to ${ }^{1} \mathrm{O}_{2}$ specially in the gpx 5 mutant. We speculated that iron might respond to the cellular redox state or deliver a redox signal to other ROS sensors.

Similarly, group C genes, which were downregulated in CC4348, were classified into subgroups. Interestingly, none of the genes were upregulated in the gpx 5 mutant (group C1); 33 genes, designated C2, were not significantly changed in the gpx 5 mutant; and 445 genes, designated $\mathrm{C} 3$, were downregulated in both gpx5 and CC4348 (Figure 5C).

The $\mathrm{C} 2$ genes encoded proteins involved in nucleotide metabolism and regulation of transcription. Transcripts of a histone H2B variant (HBV1), ATP-dependent RNA helicases (HEL12, HEL34, and HEL56), DNA-directed RNA polymerase I subunit (RPA12), and transcription initiation factor TFIID subunit 10 (Cre16.g657000) were downregulated more than 2-fold in CC4348, but remained stable in the gpx5 mutant under oxidative stress. These downregulated transcripts included some transcription factors or transcription regulators, including protein with chromosome condensation (RCC1) repeat domain (Cre12.g528350), ribosomal N-lysine methyltransferase 3 (Cre12.g541777), and leucine zipper transcription factors (Cre06.g252000 and Cre12.g490950). These transcription factors were specifically inhibited in CC4348, which might be related to GPX5 protein or low ROS concentrations.

The expression of subgroup C3 genes was downregulated both in the gpx 5 mutant and CC4348 under oxidative stress. In subgroup C3, 178 FAP transcripts were rapidly downregulated under oxidative stress in the $g p x 5$ mutant and CC 348 . These results further indicated that oxidative stress inhibits the expression of flagella-related genes, and more ROS induced deflagellation in C. reinhardtii (Figure 3D). In addition, the transcripts of four genes encoding selenoproteins, GPX1, NTR1, SELW1, and SELU1, from subgroup C3 were significantly downregulated in CC4348 and gpx5 cells under oxidative stress. The relationship between selenoproteins and oxidative stress remains to be explored.

By comparing the expression of these 1852 DEGs in CC4348 and L27, we found that there were high similar responses between CC4348 and the complementary strain L27. Among 322 upregulated DEGs in CC4348 after treatment for 30 min, roughly 79\% (253) overlapped with induced DEGs in L27. Among 386 downregulated DEGs in CC4348 after treatment for $30 \mathrm{~min}$, roughly 88\% (341) overlapped with depressed DEGs in L27. Overall, about 84\% (594 out of 708) of DEGs in CC4348 were overlapped with that in L27 after treatment for $30 \mathrm{~min}$ (Figure S6 and Table S4).

In brief, after RB treatment, CC4348 and the gpx5 mutant shared some common pathways to respond to oxidative stress showed, including upregulated ROS detoxification and ubiquitin-mediated proteolysis (subgroup A1), and inhibited the expression of cell structure related proteins and selenoproteins (subgroup C3). When GPX5 protein was deficient, some specific responses occurred, such as accelerated the TCA cycle and mETC in mitochondria to supply more ATP (subgroup B1), repressed genes related to chlorophyll metabolism and photosynthesis (subgroup A2 and A3), and downregulated iron transporters (subgroup B3).

\subsection{Effect of ${ }^{1} \mathrm{O}_{2}$ on Photosynthesis}

Previous studies on terrestrial plants and photosynthetic microorganisms have shown that hydrogen peroxide downregulates the expression of genes encoding photosynthesis-related 
proteins [45-47]. To further understand the effects of ${ }^{1} \mathrm{O}_{2}$ on the expression of photosynthesis-related genes, we analyzed the transcript changes of LHC genes. The transcript level of LHCs in CC4348 increased by two- to four-fold within $1 \mathrm{~h}$ after RB treatment. However, they fluctuated over a two-fold range during oxidative stress in the gpx5 mutant, but increased significantly in L27, even more than in CC4348 after RB treatment for 30 min (Figure 6A-C and Table S6).
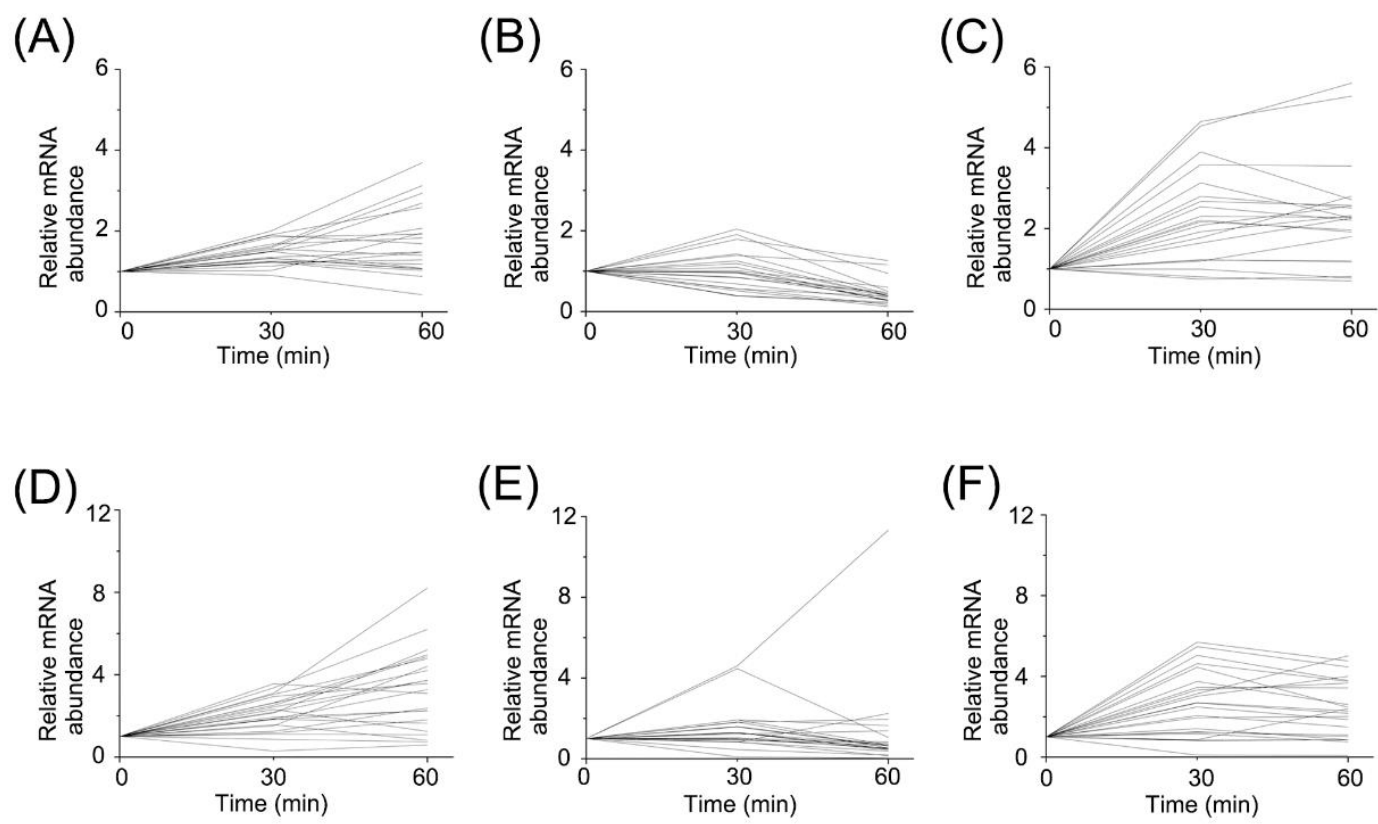

(G)

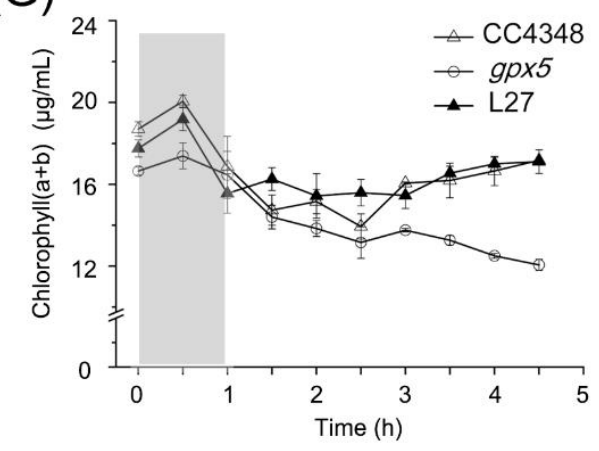

$(\mathrm{H})$

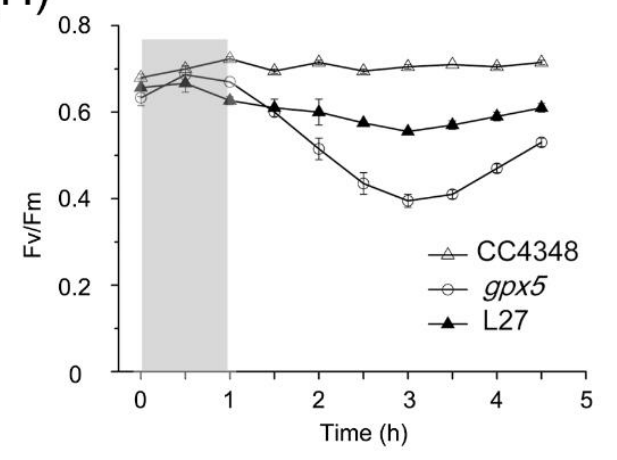

Figure 6. The response of photosystem to ${ }^{1} \mathrm{O}_{2}$. (A-C) The expression pattern of DEGs encoding light-harvesting proteins in CC4348 (A), the gpx 5 mutant (B), and L27 (C) after treated with $1 \mu \mathrm{M}$ RB determined by RNA-seq. (D-F) The expression pattern of DEGs related to chlorophyll metabolism in CC4348 (D), the gpx5 mutant (E), and L27 (F) after treated with $1 \mu \mathrm{M}$ RB determined by RNA-seq. The initial RPKM was set as 1 for each strain, separately, to normalize the data. Genes and corresponding RPKM values used in this analysis can be found in Table S6. (G,H) Comparison of the content of chlorophyll $(a+b)(\mathbf{G})$ and maximum efficiency $(\mathrm{Fv} / \mathrm{Fm})$ of photosystem II $(\mathbf{H})$ in CC4348, the gpx5 mutant and L27. The same amounts of cells were treated with $1 \mu \mathrm{M} \mathrm{RB}$ for $1 \mathrm{~h}$, and the RB was then washed out, and measurements were taken over a period of $3.5 \mathrm{~h}$. The stage of treatment is shaded with light gray.

RNA-seq data of transcripts encoding proteins involved in chlorophyll metabolism in CC4348, gpx5, and L27 strains were also analyzed at 0, 30, and $60 \mathrm{~min}$ after RB treatment (Figure 6D-F). The expression of chlorophyll-metabolism-related genes in CC4348 showed a continuous trend of upregulation within $60 \mathrm{~min}$ of oxidative stress, whereas those in the gpx 5 mutant showed an upward 
trend in expression within the first $30 \mathrm{~min}$ and remained stable in the following $30 \mathrm{~min}$, except a chlorophyll synthase gene (CHLG, Cre06.g294750) and a delta-aminolevulinic acid dehydratase gene (ALAD1, Cre02.g091050) (Figure 6E). Most of these transcripts were upregulated by less than 6-fold after $30 \mathrm{~min}$ and then remained stable abundance in L27. In CC4348, the transcript abundance of CHLG was continuously increased from 12 RPKM after RB treatment and reached a peak of 45 RPKM after $60 \mathrm{~min}$; in the gpx5 mutant, CHLG reached a peak of 11 RPKM after $30 \mathrm{~min}$ and then decreased to 3 RPKM. Although the expression of CHLG in the gpx5 mutant increased by 4.5 -fold, the overall transcript abundance was low. CHLG catalyzes the production of chlorophyll from chlorophyllide. The relative low abundance of CHLG transcript affect the final synthesis of pigments. In addition, the $A L A D 1$ transcript increased more than 6-fold (peak at 45 RPKM) after RB treatment for 60 min in CC4348, and increased about 11-fold (peak at 55 RPKM) in gpx5. The expression pattern of ALAD1 in L27 was similar with that in CC4348. ALAD1 was more strongly induced by ${ }^{1} \mathrm{O}_{2}$ than other genes in the chlorophyll synthesis pathway, suggesting that it is important for this response.

To determine the effect of oxidative stress on the photosynthetic efficiency of CC4348 and the gpx5 mutant, we measured chlorophyll pigment contents and photosynthetic efficiency every $30 \mathrm{~min}$, during $1 \mathrm{~h}$ of treatment with $1 \mu \mathrm{M}$ RB and another $3.5 \mathrm{~h}$ after the treatment was terminated. Figure $6 \mathrm{G}$ shows the total chlorophyll $a$ and $b$ contents. Consistent with the mRNA abundance of genes related to chlorophyll metabolism, the chlorophyll content of CC4348 increased by about 7\% after a 30-min treatment and then returned to $91 \%$ of the initial level after the $3.5 \mathrm{~h}$ recovery. The chlorophyll in the gpx 5 mutant increased by only $4 \%$ in the first $30 \mathrm{~min}$ of treatment but then decreased continuously after removal of $\mathrm{RB}$. The pigment content decreased to $72 \%$ of the initial state in the gpx 5 mutant at $3.5 \mathrm{~h}$ after termination of the RB treatment. The pattern of change of pigment content in L27 was similar to that of CC348, in which chlorophyll increased by $8 \%$ after $30 \mathrm{~min}$ of treatment and then decreased to $96 \%$ of the initial level. Therefore, we speculated that a low ROS concentration may stimulate the transient synthesis of chlorophyll, whereas a high ROS concentration may inhibit chlorophyll metabolism.

The maximum efficiency of PSII (Figure 6H) was also measured. The photosynthetic efficiency of PSII averaged around 0.7 in CC4348 throughout the experiment. However, at the initial time point, the maximum efficiency was only 0.63 in the gpx 5 mutant, but this increased to 0.68 after 30 min of RB treatment. At $3 \mathrm{~h}$ after the RB was removed, the maximum efficiency of PSII had declined to its lowest value, 0.4 , in the gpx5 mutant. At $3.5 \mathrm{~h}$ after the RB was removed, the maximum efficiency slowly recovered to 0.53 in the mutant, which was still lower than its initial level. The maximum efficiency of PSII of L27 was similar to that of CC348, but its value was slightly lower (about 0.6). Oxidative stress had little effect on the photosynthetic efficiency of CC348, but it had a negative effect on the gpx5 mutant. After treatment with RB, ROS accumulated in $g p x 5$ cells. Therefore, a high concentration of ROS might inhibit photosynthetic efficiency. After the removal of oxidative stress, the ROS level in cells gradually decreased and the photosynthetic efficiency recovered accordingly. These results indicated that a small amount of ROS had little effect on photosynthetic efficiency, but that a high concentration of ROS inhibited photosynthetic efficiency.

\section{Discussion}

In this study, we performed transcriptomic analyses to explore the function of GPX5 during the acclimatization to ${ }^{1} \mathrm{O}_{2}$ generated by $\mathrm{RB}$ in $\mathrm{C}$. reinhardtii. The different expression pattern of genes in algal strains with or without GPX5 function indicated that cells have specific responses to different levels of stress. Oxidative stress induced the upregulation of genes encoding proteins involved in ROS detoxification and protein degradation in these two strains. However, some genes, such as coproporphyrinogen III oxidase (CPX1), were only induced in CC4348. Another set of genes, such as a putative dTDP-glucose 4-6-dehydratase gene (SNE1), were only induced in the gpx5 mutant, in which the ROS level increased more than two-fold. We also investigated the adjustment of the transcriptional network in the gpx5 mutant at optimized laboratory growth conditions. 


\subsection{Acclimatization of the gpx5 Mutant in Laboratory Growth Conditions}

We explored the function of GPX5 under optimized laboratory growth conditions (no extra oxidative stress added). GPX5 deficiency led to a decrease of the ROS-scavenging ability in the mutants (Figure 1A). However, the gpx5 mutant can still grow under laboratory growth conditions. Functional analysis of DEGs by comparing the transcriptomes in the gpx5 mutant to CC4348 revealed that the mutant copes with the loss of GPX5 by multiple mechanisms (Figure S7). First, the synthesis of the antioxidant ASC was upregulated in the gpx5 mutant by increased abundance of the mRNA encoding VTC2, which catalyzes the first, rate-limiting step in ASC synthesis [48,49]. ASC plays an important role in protecting plants from oxidative damage [50-52], suggesting that the mutant cells might activate the ASC-dependent detoxification of ROS, thus helping to compensate for the loss of GPX5 function.

Second, the abundance of transcripts encoding redoxins, such as NRX2, NRX3, and SRX1, increased in the gpx5 mutant. These redoxins reduce disulfide bonds in proteins, to relieve cellular oxidative stress. In addition, chlorophyll synthesis declined, reducing the amount of ROS formation. In brief, under optimized laboratory growth conditions, the gpx5 mutant displayed enhanced production of antioxidants and redoxins, as well as weakened ROS production pathways to compensate for the lack of GPX5 (Figure S7).

\subsection{Adjustment of Photosystems in Response to Oxidative Stress}

Under optimized laboratory growth conditions, the transcript abundance of most of the genes encoding chlorophyll metabolism and photosynthetic antenna proteins were lower in the gpx5 mutant than in CC4348 (Figure S8). Consistent with these mRNA levels, the chlorophyll content in $g p x 5$ mutant was lower than that in CC4348 (Figure 6G). Chlorophyll is another photosensitizer; therefore, the reduction of chlorophyll contents might protect cells from more oxidative stress. After treatment with RB for $60 \mathrm{~min}$, chlorophyll-synthesis-related transcripts were upregulated, consistent with the increased content of chlorophyll after treatment in CC4348. Nevertheless, the expression of chlorophyll-synthesis-related transcripts fluctuated within 2-fold in the gpx5 mutant. The chlorophyll content of the gpx5 cells increased slightly during the first 30 min of treatment, but the increase was lower than that of CC4348. However, it decreased in the next $30 \mathrm{~min}$ of treatment and continued to decline for $3.5 \mathrm{~h}$ after RB was removed (Figure 6D,G). Since high light levels lead to robust photosynthesis but also ROS generation, the logical response of the cell is to increase chlorophyll, to better absorb the available light. When ROS is too high, photosynthesis becomes a net damaging process and must be scaled back. In a similar way, under treatment with RB, increasing ROS levels facilitated chlorophyll metabolism to physically dissipate excessive energy. When increasing ROS overwhelm the ability to detoxify, photosynthesis would be downregulated. These results suggest that short-term low levels of ROS induce a transient increase in the metabolism of chlorophyll, to better absorb the available light, but high or sustained level of ROS inhibit chlorophyll synthesis; therefore, photosynthesis becomes a net damaging process and must be scaled back.

\subsection{Regulation of Iron Homeostasis in Response to Oxidative Stress}

Among 708 DEGs, ferritin2 exhibited the highest transcript abundance, increasing 150-fold in CC4348 after a $1 \mathrm{~h}$ treatment with RB (Figure 3B,C) and increasing 338-fold in the gpx5 mutant after a 1 $\mathrm{h}$ treatment with RB. This upregulation of ferritin 2 was consistent with a previous study, in which the expression of ferritin2 increased about 80-fold in response to $\mathrm{RB}$ and 10-fold in response to $\mathrm{H}_{2} \mathrm{O}_{2}[17,44]$. By contrast, in the sak1 mutant, the transcript abundance of ferritin2 was extremely low after RB treatment (<10 RPKM) [17]. Ferritin2 is highly similar to bacterial DPS (DNA-binding protein from starved cells), which protects DNA from oxidative damage by sequestering Fe ions [53,54]. These data suggested that Fe-catalyzed DNA damage caused by ${ }^{1} \mathrm{O}_{2}$ is more serious than that caused by $\mathrm{H}_{2} \mathrm{O}_{2}$, especially in cells that lack GPX5. Besides ferritin2, transcript abundance for another two Fe-containing protein genes, SOUL1 and SOUL2, increased by more than 60-fold in CC4348 and more than 120-fold 
in the gpx 5 mutant after RB treatment for $30 \mathrm{~min}$. Increased levels of Fe-containing proteins would chelate more Fe to reduce Fe-catalyzed DNA damage.

In addition to Fe chelation, Fe mobilization and Fe assimilation played important roles in Fe homeostasis. Chlamydomonas has two Fe-uptake pathways, the high-affinity pathway, involving the ferroxidase FOX1 coupled to a ferric ion transporter FTR1, and the lower-affinity pathway, involving inducible ZIP family transporters IRT1 and IRT2 [55,56]. Iron assimilation components also include ferrireductase (FRE1) and Fe-assimilating protein FEA1 (Cre12.g546550). FEA1 is a secreted protein that facilitates high-affinity iron uptake, perhaps by concentrating iron in the vicinity of the cell [55]. The transcripts of all iron mobilization and iron assimilation components, including FOX1, FTR1, IRT2, and FRE1, did not significantly change in CC4348, except FEA1, which was upregulated about 3-fold after RB treatment for $1 \mathrm{~h}$. However, all of these components decreased in transcript abundance in the gpx 5 mutant. These meant that lowering the concentration of Fe by chelation and mobilization likely enhances the ability of Chlamydomonas to tolerate oxidative stress.

\subsection{The Effect of ${ }^{1} \mathrm{O}_{2}$ on the Expression of Selenoproteins}

Although selenocysteine has stronger reducibility than cysteine, selenoproteins with selenocysteine in the active site were not all induced by oxidative stress in our study. Twelve transcripts of selenoproteins were detected in C. reinhardtii cells, including nine downregulated and three upregulated transcripts (Figure S9). Among them, two selenocysteine glutathione peroxidases, GPX1 and GPX2, are involved in the degradation of hydrogen peroxide and belong to the same family as GPX5. However, these two transcripts showed an unexpected downregulation, in contrast to that of GPX5, in both strains. Although not significantly changed, the transcript abundance of GPX2 decreased slightly in CC4348 and the gpx 5 mutant. NADPH-dependent thioredoxin reductase (NTR) has been reported to play a role in abiotic stress responses in Arabidopsis [57]. In CC4348 cells, the transcripts encoding selenium-containing NTR1 (Cre08.g368400) were downregulated, while transcripts of the gene encoding selenium-free NTR2 (Cre02.g098850) were upregulated after the addition of RB. In addition, the transcript abundance of the selenium-binding protein gene SBD1 (Cre03.g166050) significantly increased under oxidative stress in both CC4348 and gpx5 cells. The Lotus japonicus homolog of SBD1 is thought to have more than one physiological role and has been implicated in controlling the oxidation/reduction status of target proteins in vesicular Golgi transport [58]. In summary, oxidative stress influenced the expression of some selenoproteins, but the underlying mechanism remains to be further studied.

In addition to members of the GPX and NTR families, the members of other protein families showed different expression patterns during oxidative stress. For example, NRX1 did not significantly change in CC4348, but it increased in gpx5, in contrast to the pattern of NRX2. TRX21 and SELU1 from the thioredoxin superfamily were downregulated in CC4348 and $g p x 5$ under oxidative stress, while another 8 members were upregulated (LCI7, PDI1, PDI2, PDI4, PDIA6, TRX5, NRX2, and Endoplasmic Reticulum-Golgi Intermediate Compartment (ERGIC, Cre08.g358579)). These observations indicated that even members of the same protein family function independently.

\subsection{GPX5 is Necessary for ${ }^{1} \mathrm{O}_{2}$ Acclimation in C. reinhardtii}

Two proteins responsible for the response to ${ }^{1} \mathrm{O}_{2}$, the $\mathrm{C} 2 \mathrm{H} 2$ zinc finger protein MBS (Cre09.g416500) [16] and the putative transcription factor SAK1 (Cre17.g741300) [17], are located in the cytosol, as is the GPX5 protein [15]. Therefore, we wondered about the relationship among these three proteins in ${ }^{1} \mathrm{O}_{2}$ signaling. The ${ }^{1} \mathrm{O}_{2}$-responsive reporter gene (HPS70A) was not induced by $\mathrm{RB}$ in the mbs or the sak1 mutant. In the gpx 5 mutant, the transcript abundance of HSP70A increased by 12-fold compared with CC4348. So, SAK1 and MBS might function in the same signaling pathway, but GPX5 does not function in this pathway. Furthermore, the expression of MBS was not affected after RB treatment in the sak1 mutant, so the MBS protein likely functions upstream of SAK1 in the response to ${ }^{1} \mathrm{O}_{2}$. 
The transcriptome of CC348 after treatment with $1 \mu \mathrm{M}$ RB for $1 \mathrm{~h}$ was compared to the transcriptome of $4 \mathrm{~A}+$, the parental strain of the sak1 mutant, with the same treatment. Among 402 upregulated genes in CC4348, 95 were also upregulated in 4A+. These transcripts encoded redox-related proteins and ATP-binding cassette (ABC) transporters, as well as GPX5 and SAK1. GPX5 was upregulated 17-fold in CC4348 and 14-fold in 4A+ after treatment with $1 \mu \mathrm{M}$ RB. Similarly, SAK1 was upregulated 6-fold in CC4348 and 8-fold in $4 \mathrm{~A}+$ in the same conditions. Some other strongly ${ }^{1} \mathrm{O}_{2}$-responsive genes also showed the same pattern, such as SOUL1, SOUL2, CFA1, CFA2, LCI7, and VTC2. These data suggest that our transcriptome data are reliable.

Compared the transcriptomics data from the gpx5 mutant and the sak1 mutant subjected to oxidative stress treatment using $1 \mu \mathrm{M}$ RB for $1 \mathrm{~h}$, there were 456 overlap DEGs which were induced in both the gpx5 mutant and the sak1 mutant, while 106 overlap DEGs depressed. When C. reinhardtii cells were treated with RB, the TCA cycle was induced by oxidative stress in the gpx5 mutant, whereas these changes were absent in the sak1 mutant. We also detected numerous transcripts encoding cell structure proteins, including FAPs, which were downregulated after RB treatment in the gpx5 mutant. However, some of them increased in abundance in the sak1 mutant after RB treatment. For example, the transcripts encoding alpha tubulin (TUA1 and TUA2) and beta tubulin (TUB1 and TUB2) were downregulated in CC348 and gpx5 after RB treatment, but they showed no significant change and high abundances (>500 RPKM). Other factors might affect cell structure proteins besides oxidative stress.

We identified the SAK1 transcript in the $g p x 5$, control, and complemented strains. After treatment with $1 \mu \mathrm{M}$ RB for $1 \mathrm{~h}$, the SAK1 transcript increased in abundance, from 6 to 36 RPKM in CC4348, from 6 to 107 RPKM in the gpx5 mutant, and from 14 to 179 RPKM in complemented strain L27. We also found the GPX5 transcript in the sak1 mutant from the published data [17], which increased in abundance, from 314 to 1112 RPKM. These data indicated that SAK1 protein and GPX5 protein did not function in the same pathway, and the expression of cell structure proteins may be regulated separately. We also observed similarities and differences in the response to ${ }^{1} \mathrm{O}_{2}$ and $\mathrm{H}_{2} \mathrm{O}_{2}$. After $\mathrm{RB}$ treatment, 18 transcripts involved in chlorophyll biosynthesis were upregulated in CC4348, most of which remained unchanged in the gpx 5 mutant and sak1 mutant. By contrast, almost all of these were downregulated after $\mathrm{H}_{2} \mathrm{O}_{2}$ treatment [44]. During $1 \mathrm{~h}$ of $\mathrm{RB}$ treatment, the increased photosystem transcripts did not have enough time to elevate the photosynthetic efficiency in CC4348. Transiently increased photosynthetic efficiency was observed in the gpx5 mutant. After cells were treated with $\mathrm{H}_{2} \mathrm{O}_{2}$, the photosynthetic efficiency slowed down in the first $0.5 \mathrm{~h}$ and returned after $4 \mathrm{~h}$. Maybe the initial burst of ${ }^{1} \mathrm{O}_{2}$ stimulated the photosystem, while $\mathrm{H}_{2} \mathrm{O}_{2}$ had a negative impact on photosynthetic rate. Therefore, ${ }^{1} \mathrm{O}_{2}$ and $\mathrm{H}_{2} \mathrm{O}_{2}$ affect cell physiology in different ways.

\subsection{The Genome-Wide Response to ${ }^{1} \mathrm{O}_{2}$ in gpx5 Mutants}

By comparing the transcriptomes of the gpx 5 mutant and CC348 after RB treatment, we examined the specific responses to different levels of oxidative stress. When CC4348 was treated with RB, the ROS level increased only 1-fold. Under this condition, antioxidant enzymes, such as GPX family proteins, and the biosynthesis of the antioxidant ASC were induced. The chlorophyll contents increased and carbon metabolism, including glycolysis and the OPP pathway, were upregulated. The abundance of ferritin2 was strongly increased to chelate more iron, thus protecting biomacromolecules from oxidation by iron. At the same time, the iron transporters were unchanged in abundance, and FEA1 was upregulated, to avoid introducing extracellular iron into cells. In addition, transcripts encoding selenoproteins and cell structural proteins, especially FAPs, were significantly downregulated under oxidative stress (Figure 7A). 

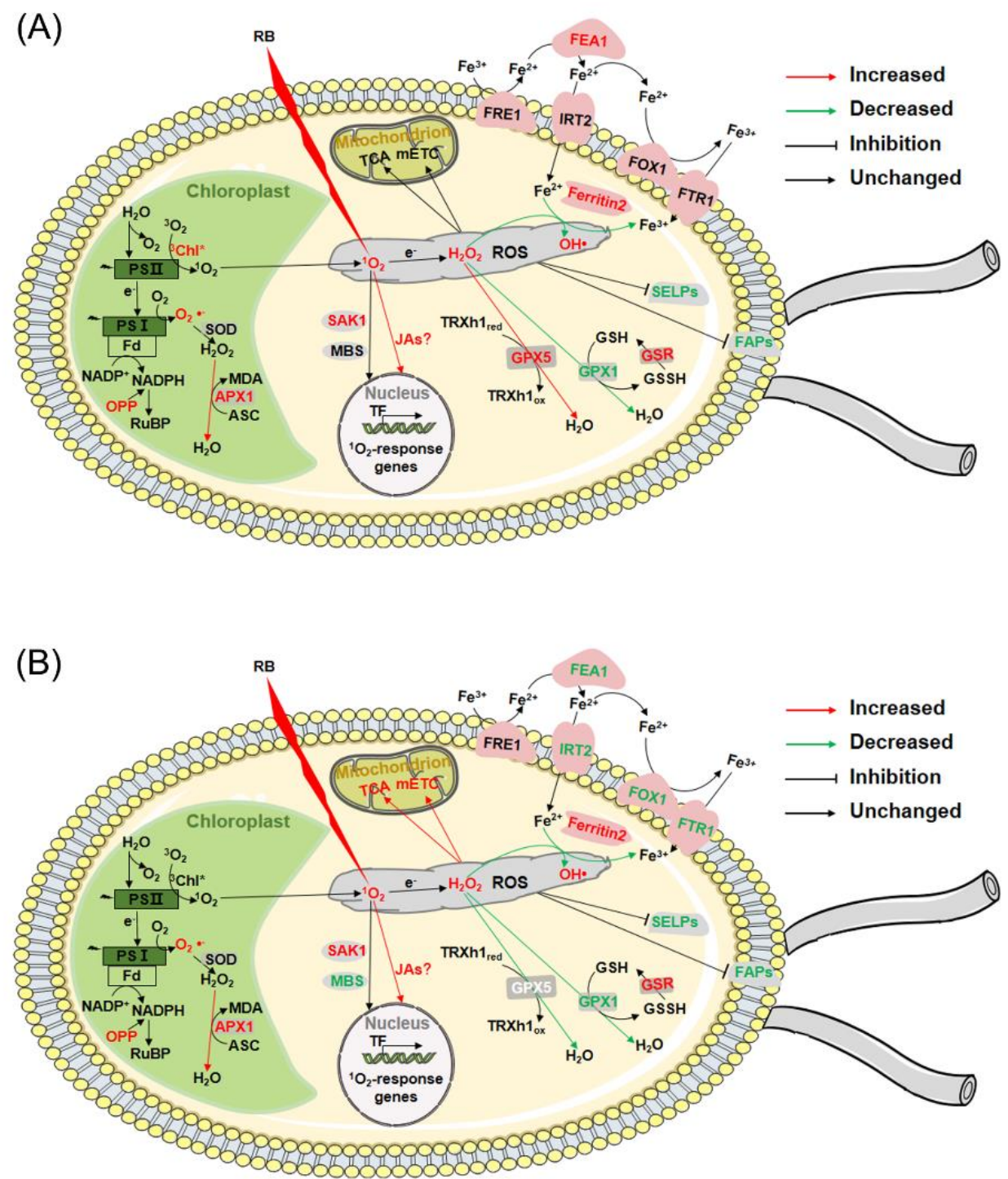

Figure 7. Summary of the cellular response to oxidative stress in CC4348 (A) and the gpx5 mutant (B). (A) After treatment with RB, the CC348 cells upregulated the ROS detoxification system and inhibited the expression of flagellar associated proteins (FAPs) and selenoproteins (SELPs). (B) When the gpx 5 mutant suffered the treatment with RB, expect for the same responses of ROS detoxification system, FAPs and SELPs, several specific responses occurred, such as accelerated the TCA cycle and mETC in mitochondria, repressed genes related to chlorophyll metabolism and photosynthesis, and downregulated iron transporters. Red characters indicate upregulated expression of related proteins or metabolic pathways after RB treatment, while green characters indicate downregulated expression of related proteins or metabolic pathways after RB treatment. Abbreviations: APX, ascorbate peroxidase; ASC, ascorbate; FAPs, flagellar associated proteins; Fd, ferredoxin; FEA1, Fe-assimilating protein 1; FOX1, ferroxidase; FRE, ferrireductase 1; FTR1, ferric ion transporter 1; GPX, glutathione peroxidase; GSR, glutathione reductase; GSH, glutathione; GSSG, oxidized glutathione; IRT2, ferrous ion transporter 2; mETC, mitochondrial electron transfer chain; OPPP, oxidative pentose phosphate pathway; ROS, reactive oxygen species; PRX, peroxiredoxin; PSI/II, photosystem I/II; RB, rose bengal; RuBP, ribulose 1,5-bisphosphate; SELPs, selenoproteins; SOD, superoxide dismutase; TF, transcription factor; TRXh1, thioredoxin h1. SAK1 [17] and MBS [16] are two cytosolic proteins required for induction of nuclear gene expression responses by ${ }^{1} \mathrm{O}_{2}$.

Cells lacking GPX5 were in severe oxidative stress after being subjected to RB treatment, and their ROS levels increased up to 3-fold. Under this condition, the GPX5-mediated pathway to detoxify into 
$\mathrm{H}_{2} \mathrm{O}_{2}$ was defective, resulting in high levels of ROS. In addition to the shared responses with CC4348 after RB treatment, some specific responses occurred in the gp $x 5$ mutant. Continuous high levels of ROS repressed genes related to chlorophyll metabolism and photosynthesis. Serious oxidative stress accelerated the TCA cycle and mETC in mitochondria to supply more ATP. Ferritin2 was also induced, and iron transporters were downregulated, to enhance the protection from damage by iron. However, FEA1 was downregulated. At high levels of ROS, SAK1 was still increased in abundance, but MBS decreased in the gp $x 5$ mutant after treatment. By using multiple adaption mechanisms, cells managed to survive and recover from oxidative stress (Figure 7B).

In summary, this study revealed that GPX5 plays a crucial role in maintaining ROS homeostasis in cells and mediating the dose-dependent response to ROS stress in Chlamydomonas. The function of GPX5 cannot be replaced completely by other ROS-scavenging enzymes or systems. Iron homeostasis may play an important role in acclimatization to oxidative stress.

Supplementary Materials: The following additional supporting information are available online. Figure S1: The good correlation between the cell number and OD750. Figure S2: The diagram for experimental design and analysis. Figure S3: A comparison of fold change (log2) values of transcript levels measured by RNA-Seq and qRT-PCR. Figure S4: The effect of $1 \mathrm{O} 2$ on flagella. Figure S5: Expression pattern of differentially expressed genes (DEGs) related to proteasomes. Figure S6: Expression pattern of the parental strain CC4348 and the complementary strain L27 after treatment with $1 \mu \mathrm{M}$ RB for $30 \mathrm{~min}$. Figure S7: Production and scavenging of ROS in the gpx5 mutant under standard growth condition. Figure S8: Expression pattern of differentially expressed genes (DEGs) related to chlorophyll metabolism and photosynthesis. Figure S9: The transcript abundance of genes encoding selenoproteins. Table S1: Expression estimates for all genes in CC4348, gpx5, and L27. Table S2: Genes whose mRNA abundance significantly changed in the gpx 5 mutant under optimized growth conditions. Table S3: Genes whose mRNA abundance significantly changed in CC4348 after treatment with $1 \mu \mathrm{M}$ RB for 30 min. Table S4: Genes whose mRNA abundance significantly changed in CC4348 or gpx5 mutant after treatment with $1 \mu \mathrm{M}$ RB for $30 \mathrm{~min}$. Table S5: mRNA abundances for genes encoding proteins relating to ubiquitin-proteasome system. Table S6: mRNA abundances for genes encoding proteins relating to chlorophyll metabolism and light-harvesting proteins. Table S7: Forward and reverse primers of genes used in RT-qPCR.

Author Contributions: Conceptualization, K.H. and X.-Q.X.; data curation, X.M.; formal analysis, X.M. and W.Z.; funding acquisition, K.H. and X.-Q.X.; investigation, B.Z. and Y.C.; methodology, X.M., B.Z., and R.M.; resources, R.M. and Y.C.; software, X.M. and Y.D.; supervision, K.H. and X.-Q.X.; validation, M.S.; visualization, R.M. and X.D.; writing-original draft, X.M. and B.Z.; writing-review and editing, K.H. and X.-Q.X. All authors have read and agreed to the published version of the manuscript.

Funding: This study was supported by the Major Research Plan of the National Natural Science Foundation of China (Grant No. 91851201) and the 100-talent Program, the Chinese Academy of Sciences (Grant No. Y22302). The authors would also like to thank Weyland Cheng for his assistance in editing the manuscript.

Acknowledgments: We would like to thank the Wuhan Branch, Supercomputing Center, Chinese Academy of Sciences for his assistance in processing data.

Conflicts of Interest: The authors declare that they have no conflict of interest.

\section{References}

1. Sies, H.; Berndt, C.; Jones, D.P. Oxidative Stress. Annu. Rev. Biochem. 2017, 86, 715-748. [CrossRef] [PubMed]

2. Del Rio, L.A. ROS and RNS in plant physiology: An overview. J. Exp. Bot. 2015, 66, 2827-2837. [CrossRef] [PubMed]

3. Foyer, C.H. Reactive oxygen species, oxidative signaling and the regulation of photosynthesis. Environ. Exp. Bot. 2018, 154, 134-142. [CrossRef] [PubMed]

4. Apel, K.; Hirt, H. Reactive oxygen species: Metabolism, oxidative stress, and signal transduction. Annu. Rev. Plant Biol. 2004, 55, 373-399. [CrossRef] [PubMed]

5. Mittler, R. ROS Are Good. Trends Plant Sci. 2017, 22, 11-19. [CrossRef]

6. Foyer, C.H.; Noctor, G. Redox signaling in plants. Antioxid. Redox Signal. 2013, 18, 2087-2090. [CrossRef]

7. Konig, J.; Muthuramalingam, M.; Dietz, K.J. Mechanisms and dynamics in the thiol/disulfide redox regulatory network: Transmitters, sensors and targets. Curr. Opin. Plant Biol. 2012, 15, 261-268. [CrossRef]

8. Mignolet-Spruyt, L.; Xu, E.; Idanheimo, N.; Hoeberichts, F.A.; Muhlenbock, P.; Brosche, M.; Van Breusegem, F.; Kangasjarvi, J. Spreading the news: Subcellular and organellar reactive oxygen species production and signalling. J. Exp. Bot. 2016, 67, 3831-3844. [CrossRef] 
9. Mittler, R.; Vanderauwera, S.; Suzuki, N.; Miller, G.; Tognetti, V.B.; Vandepoele, K.; Gollery, M.; Shulaev, V.; Van Breusegem, F. ROS signaling: The new wave? Trends Plant Sci. 2011, 16, 300-309. [CrossRef]

10. Vaahtera, L.; Brosche, M.; Wrzaczek, M.; Kangasjarvi, J. Specificity in ROS signaling and transcript signatures. Antioxid. Redox Signal. 2014, 21, 1422-1441. [CrossRef]

11. Leisinger, U.; Rüfenacht, K.; Fischer, B.; Pesaro, M.; Spengler, A.; Zehnder, A.J.; Eggen, R.I. The glutathione peroxidase homologous gene from Chlamydomonas reinhardtii is transcriptionally up-regulated by singlet oxygen. Plant Mol. Biol. 2001, 46, 395-408. [CrossRef]

12. Fischer, B.B.; Dayer, R.; Schwarzenbach, Y.; Lemaire, S.D.; Behra, R.; Liedtke, A.; Eggen, R.I. Function and regulation of the glutathione peroxidase homologous gene GPXH/GPX5 in Chlamydomonas reinhardtii. Plant Mol. Biol. 2009, 71, 569-583. [CrossRef] [PubMed]

13. Fischer, B.B.; Krieger-Liszkay, A.; Eggen, R.I.L. Oxidative stress induced by the photosensitizers neutral red (type I) or rose bengal (type II) in the light causes different molecular responses in Chlamydomonas Reinhardtii. Plant Sci. 2005, 168, 747-759. [CrossRef]

14. Fischer, B.B.; Krieger-Liszkay, A.; Hideg, E.; Snyrychova, I.; Wiesendanger, M.; Eggen, R.I. Role of singlet oxygen in chloroplast to nucleus retrograde signaling in Chlamydomonas reinhardtii. FEBS Lett. 2007, 581, 5555-5560. [CrossRef] [PubMed]

15. Miao, R.; Ma, X.; Deng, X.; Huang, K. High level of reactive oxygen species inhibits triacylglycerols accumulation in Chlamydomonas reinhardtii. Algal Res. 2019, 38, 101400. [CrossRef]

16. Shao, N.; Duan, G.Y.; Bock, R. A mediator of singlet oxygen responses in Chlamydomonas reinhardtii and Arabidopsis identified by a luciferase-based genetic screen in algal cells. Plant Cell 2013, 25, 4209-4226. [CrossRef]

17. Wakao, S.; Chin, B.L.; Ledford, H.K.; Dent, R.M.; Casero, D.; Pellegrini, M.; Merchant, S.S.; Niyogi, K.K. Phosphoprotein SAK1 is a regulator of acclimation to singlet oxygen in Chlamydomonas reinhardtii. Elife 2014, 3, e02286. [CrossRef]

18. Zabawinski, C.; Van Den Koornhuyse, N.; D’Hulst, C.; Schlichting, R.; Giersch, C.; Delrue, B.; Lacroix, J.M.; Preiss, J.; Ball, S. Starchless mutants of Chlamydomonas reinhardtii lack the small subunit of a heterotetrameric ADP-glucose pyrophosphorylase. J. Bacteriol. 2001, 183, 1069-1077. [CrossRef]

19. Wang, Z.T.; Ullrich, N.; Joo, S.; Waffenschmidt, S.; Goodenough, U. Algal lipid bodies: Stress induction, purification, and biochemical characterization in wild-type and starchless Chlamydomonas Reinhardtii. Eukaryot. Cell 2009, 8, 1856-1868. [CrossRef]

20. Blaby, I.K.; Glaesener, A.G.; Mettler, T.; Fitz-Gibbon, S.T.; Gallaher, S.D.; Liu, B.; Boyle, N.R.; Kropat, J.; Stitt, M.; Johnson, S.; et al. Systems-level analysis of nitrogen starvation-induced modifications of carbon metabolism in a Chlamydomonas reinhardtii starchless mutant. Plant Cell 2013, 25, 4305-4323. [CrossRef]

21. Tran, Q.G.; Cho, K.; Park, S.B.; Kim, U.; Lee, Y.J.; Kim, H.S. Impairment of starch biosynthesis results in elevated oxidative stress and autophagy activity in Chlamydomonas reinhardtii. Sci. Rep. 2019, 9, 9856. [CrossRef] [PubMed]

22. Harris, E.H. The Chlamydomonas Sourcebook: Introduction to Chlamydomonas and Its Laboratory Use; Elsevier LTD: Oxford, UK, 2009; Volume 1.

23. Patel, R.K.; Jain, M. NGS QC Toolkit: A toolkit for quality control of next generation sequencing data. PLoS ONE 2012, 7, e30619. [CrossRef]

24. Trapnell, C.; Pachter, L.; Salzberg, S.L. TopHat: Discovering splice junctions with RNA-Seq. Bioinformatics 2009, 25, 1105-1111. [CrossRef] [PubMed]

25. Anders, S.; Pyl, P.T.; Huber, W. HTSeq-a Python framework to work with high-throughput sequencing data. Bioinformatics 2015, 31, 166-169. [CrossRef]

26. Robinson, M.D.; McCarthy, D.J.; Smyth, G.K. edgeR: A Bioconductor package for differential expression analysis of digital gene expression data. Bioinformatics 2010, 26, 139-140. [CrossRef] [PubMed]

27. Mortazavi, A.; Williams, B.A.; McCue, K.; Schaeffer, L.; Wold, B. Mapping and quantifying mammalian transcriptomes by RNA-Seq. Nat. Methods 2008, 5, 621-628. [CrossRef] [PubMed]

28. Livak, K.J.; Schmittgen, T.D. Analysis of relative gene expression data using real-time quantitative PCR and the 2- $\Delta \Delta$ CT method. Methods 2001, 25, 402-408. [CrossRef]

29. Yoshida, K.; Igarashi, E.; Wakatsuki, E.; Miyamoto, K.; Hirata, K. Mitigation of osmotic and salt stresses by abscisic acid through reduction of stress-derived oxidative damage in Chlamydomonas Reinhardtii. Plant Sci. 2004, 167, 1335-1341. [CrossRef] 
30. Blaby, I.K.; Blaby-Haas, C.E.; Tourasse, N.; Hom, E.F.; Lopez, D.; Aksoy, M.; Grossman, A.; Umen, J.; Dutcher, S.; Porter, M.; et al. The Chlamydomonas genome project: A decade on. Trends Plant Sci. 2014, 19, 672-680. [CrossRef]

31. Merchant, S.S.; Prochnik, S.E.; Vallon, O.; Harris, E.H.; Karpowicz, S.J.; Witman, G.B.; Terry, A.; Salamov, A.; Fritz-Laylin, L.K.; Marechal-Drouard, L.; et al. The Chlamydomonas genome reveals the evolution of key animal and plant functions. Science 2007, 318, 245-250. [CrossRef]

32. Thimm, O.; Bläsing, O.; Gibon, Y.; Nagel, A.; Meyer, S.; Krüger, P.; Selbig, J.; Müller, L.A.; Rhee, S.Y.; Stitt, M. Mapman: A user-driven tool to display genomics data sets onto diagrams of metabolic pathways and other biological processes. Plant J. 2004, 37, 914-939. [CrossRef] [PubMed]

33. Lopez, D.; Casero, D.; Cokus, S.J.; Merchant, S.S. Algal Functional Annotation Tool: A web-based analysis suite to functionally interpret large gene lists using integrated annotation and expression data. BMC Bioinform. 2011, 12, 282. [CrossRef] [PubMed]

34. Urzica, E.I.; Adler, L.N.; Page, M.D.; Linster, C.L.; Arbing, M.A.; Casero, D.; Pellegrini, M.; Merchant, S.S.; Clarke, S.G. Impact of oxidative stress on ascorbate biosynthesis in Chlamydomonas via regulation of the VTC2 gene encoding a GDP-L-galactose phosphorylase. J. Biol. Chem. 2012, 287, 14234-14245. [CrossRef] [PubMed]

35. Dixon, D.P.; Hawkins, T.; Hussey, P.J.; Edwards, R. Enzyme activities and subcellular localization of members of the Arabidopsis glutathione transferase superfamily. J. Exp. Bot. 2009, 60, 1207-1218. [CrossRef] [PubMed]

36. Mueller, L.A.; Goodman, C.D.; Silady, R.A.; Walbot, V. AN9, a petunia glutathione S-transferase required for anthocyanin sequestration, is a flavonoid-binding protein. Plant Physiol. 2000, 123, 1561-1570. [CrossRef]

37. Kampranis, S.C.; Damianova, R.; Atallah, M.; Toby, G.; Kondi, G.; Tsichlis, P.N.; Makris, A.M. A novel plant glutathione S-transferase/peroxidase suppresses Bax lethality in yeast. J. Biol. Chem. 2000, 275, 29207-29216. [CrossRef]

38. Cummins, I.; Cole, D.J.; Edwards, R. A role for glutathione transferases functioning as glutathione peroxidases in resistance to multiple herbicides in black-grass. Plant J. 1999, 18, 285-292. [CrossRef]

39. Sun, W.; Xu, X.; Zhu, H.; Liu, A.; Liu, L.; Li, J.; Hua, X. Comparative transcriptomic profiling of a salt-tolerant wild tomato species and a salt-sensitive tomato cultivar. Plant Cell Physiol. 2010, 51, 997-1006. [CrossRef]

40. Galle, A.; Csiszar, J.; Secenji, M.; Guoth, A.; Cseuz, L.; Tari, I.; Gyorgyey, J.; Erdei, L. Glutathione transferase activity and expression patterns during grain filling in flag leaves of wheat genotypes differing in drought tolerance: Response to water deficit. J. Plant Physiol. 2009, 166, 1878-1891. [CrossRef]

41. Wada, H.; Gombos, Z.; Murata, N. Enhancement of chilling tolerance of a cyanobacterium by genetic manipulation of fatty acid desaturation. Nature 1990, 347, 200-203. [CrossRef]

42. Piotrowska-Niczyporuk, A.; Bajguz, A.; Zambrzycka, E.; Godlewska-Zylkiewicz, B. Phytohormones as regulators of heavy metal biosorption and toxicity in green alga Chlorella vulgaris (Chlorophyceae). Plant Physiol. Biochem. 2012, 52, 52-65. [CrossRef] [PubMed]

43. Hu, Y.; Jiang, Y.; Han, X.; Wang, H.; Pan, J.; Yu, D. Jasmonate regulates leaf senescence and tolerance to cold stress: Crosstalk with other phytohormones. J. Exp. Bot. 2017, 68, 1361-1369. [CrossRef] [PubMed]

44. Blaby, I.K.; Blaby-Haas, C.E.; Perez-Perez, M.E.; Schmollinger, S.; Fitz-Gibbon, S.; Lemaire, S.D.; Merchant, S.S. Genome-wide analysis on Chlamydomonas reinhardtii reveals the impact of hydrogen peroxide on protein stress responses and overlap with other stress transcriptomes. Plant J. 2015, 84, 974-988. [CrossRef] [PubMed]

45. Vandenabeele, S.; Van Der Kelen, K.; Dat, J.; Gadjev, I.; Boonefaes, T.; Morsa, S.; Rottiers, P.; Slooten, L.; Van Montagu, M.; Zabeau, M.; et al. A comprehensive analysis of hydrogen peroxide-induced gene expression in tobacco. Proc. Natl. Acad. Sci. USA 2003, 100, 16113-16118. [CrossRef] [PubMed]

46. Desikan, R.; Soheila, A.H.; Hancock, J.T.; Neill, S.J. Regulation of the Arabidopsis Transcriptome by Oxidative Stress. Plant Physiol. 2001, 127, 159-172. [CrossRef]

47. Zeller, T.; Moskvin, O.V.; Li, K.; Klug, G.; Gomelsky, M. Transcriptome and physiological responses to hydrogen peroxide of the facultatively phototrophic bacterium Rhodobacter sphaeroides. J. Bacteriol. 2005, 187, 7232-7242. [CrossRef]

48. Linster, C.L.; Gomez, T.A.; Christensen, K.C.; Adler, L.N.; Young, B.D.; Brenner, C.; Clarke, S.G. Arabidopsis VTC2 encodes a GDP-L-galactose phosphorylase, the last unknown enzyme in the Smirnoff-Wheeler pathway to ascorbic acid in plants. J. Biol. Chem. 2007, 282, 18879-18885. [CrossRef] 
49. Laing, W.A.; Wright, M.A.; Cooney, J.; Bulley, S.M. The missing step of the L-galactose pathway of ascorbate biosynthesis in plants, an L-galactose guanyltransferase, increases leaf ascorbate content. Proc. Natl. Acad. Sci. USA 2007, 104, 9534-9539. [CrossRef]

50. Smirnoff, N. Ascorbate biosynthesis and function in photoprotection. Philos. Trans. R. Soc. Lond. B 2000, 355, 1455-1464. [CrossRef]

51. Smirnoff, N.; Wheeler, G.L. Ascorbic acid in plants: Biosynthesis and function. Crit. Rev. Biochem. Mol. Biol. 2000, 35, 291-314. [CrossRef]

52. Gill, S.S.; Tuteja, N. Reactive oxygen species and antioxidant machinery in abiotic stress tolerance in crop plants. Plant Physiol. Biochem. 2010, 48, 909-930. [CrossRef] [PubMed]

53. Almiron, M.; Link, A.J.; Furlong, D.; Kolter, R. A novel DNA-binding protein with regulatory and protective roles in starved Escherichia coli. Genes Dev. 1992, 6, 2646-2654. [CrossRef] [PubMed]

54. Pulliainen, A.T.; Kauko, A.; Haataja, S.; Papageorgiou, A.C.; Finne, J. Dps/Dpr ferritin-like protein: Insights into the mechanism of iron incorporation and evidence for a central role in cellular iron homeostasis in Streptococcus suis. Mol. Microbiol. 2005, 57, 1086-1100. [CrossRef] [PubMed]

55. Allen, M.D.; del Campo, J.A.; Kropat, J.; Merchant, S.S. FEA1, FEA2, and FRE1, encoding two homologous secreted proteins and a candidate ferrireductase, are expressed coordinately with FOX1 and FTR1 in iron-deficient Chlamydomonas reinhardtii. Eukaryot. Cell 2007, 6, 1841-1852. [CrossRef]

56. Chen, J.C.; Hsieh, S.I.; Kropat, J.; Merchant, S.S. A ferroxidase encoded by FOX1 contributes to iron assimilation under conditions of poor iron nutrition in Chlamydomonas. Eukaryot. Cell 2008, 7, 541-545. [CrossRef]

57. Serrato, A.J.; Pérez-Ruiz, J.M.; Spínola, M.C.; Cejudo, F.J. A novel NADPH thioredoxin reductase, localized in the chloroplast, which deficiency causes hypersensitivity to abiotic stress in Arabidopsis thaliana. J. Biol. Chem. 2004, 279, 43821-43827. [CrossRef]

58. Flemetakis, E.; Agalou, A.; Kavroulakis, N.; Dimou, M.; Martsikovskaya, A.; Slater, A.; Spaink, H.P.; Roussis, A.; Katinakis, P. Lotus japonicus gene Ljsbp is highly conserved among plants and animals and encodes a homologue to the mammalian selenium-binding proteins. Mol. Plant-Microbe Interact. 2002, 15, 313-322. [CrossRef]

(C) 2020 by the authors. Licensee MDPI, Basel, Switzerland. This article is an open access article distributed under the terms and conditions of the Creative Commons Attribution (CC BY) license (http://creativecommons.org/licenses/by/4.0/). 